\title{
Convex potentials and optimal shift generated oblique duals in shift invariant spaces
}

\author{
María J. Benac *, Pedro G. Massey *, and Demetrio Stojanoff * ${ }^{\dagger}$ \\ Depto. de Matemática, FCE-UNLP and IAM-CONICET, Argentina
}

\begin{abstract}
We introduce extensions of the convex potentials for finite frames (e.g. the frame potential defined by Benedetto and Fickus) in the framework of Bessel sequences of integer translates of finite sequences in $L^{2}\left(\mathbb{R}^{k}\right)$. We show that under a natural normalization hypothesis, these convex potentials detect tight frames as their minimizers. We obtain a detailed spectral analysis of the frame operators of shift generated oblique duals of a fixed frame of translates. We use this result to obtain the spectral and geometrical structure of optimal shift generated oblique duals with norm restrictions, that simultaneously minimize every convex potential; we approach this problem by showing that the water-filling construction in probability spaces is optimal with respect to submajorization (within an appropriate set of functions) and by considering a non-commutative version of this construction for measurable fields of positive operators.
\end{abstract}

AMS subject classification: $42 \mathrm{C} 15$.

Keywords: frames of translates, shift invariant subspaces, oblique duality, majorization, convex potentials.

\footnotetext{
${ }^{*}$ Partially supported by CONICET (PIP 0435/10) and Universidad Nacional de La PLata (UNLP 11X681)

†e-mail addresses: mjbenac@gmail.com , massey@mate.unlp.edu.ar ,demetrio@mate.unlp.edu.ar
} 


\section{Contents}

1 Introduction $\quad 2$

2 Preliminaries 4

2.1 Frames for subspaces and oblique duality . . . . . . . . . . . 4

2.2 Shift-invariant subspaces and frames of translates . . . . . . . . . . . 6

2.3 Majorization in probability spaces . . . . . . . . . . . . . 7

3 Convex potentials for sequences of translates in FSI spaces 9

4 Fine spectral structure of shift generated oblique duals in FSI subspaces 14

4.1 SG oblique duals and aliasing in FSI subspaces . . . . . . . . . . . . . 14

4.2 Fine spectral structure of SG oblique duals . . . . . . . . . . . . . . 16

5 Applications: optimal oblique SG-duals with norm restrictions 19

5.1 Water-filling in measure spaces . . . . . . . . . . . . . . . . . 20

5.2 Optimal SG-duals with norm restrictions: NC water-filling . . . . . . . . . . . . 22

6 Appendix: spectral structure of $U_{\mathcal{V}}(E(\mathcal{G})) \quad \mathbf{2 7}$

\section{Introduction}

Let $\mathcal{W}$ be a closed subspace of a separable complex Hilbert space $\mathcal{H}$ and let $\mathbb{I}$ be a finite or countable infinite set. A frame $\mathcal{F}=\left\{f_{i}\right\}_{i \in \mathbb{I}}$ for $\mathcal{W}$ allows for linear (typically redundant) and stable encodingdecoding schemes of vectors (signals) in $\mathcal{W}$ (see Section 2.1 for definitions and technical results). Indeed, if $\mathcal{V}$ is a closed subspace of $\mathcal{H}$ such that $\mathcal{V} \oplus \mathcal{W}^{\perp}=\mathcal{H}($ e.g. $\mathcal{V}=\mathcal{W})$ then it is possible to find frames $\mathcal{G}=\left\{g_{i}\right\}_{i \in \mathbb{I}}$ for $\mathcal{V}$ such that

$$
f=\sum_{i \in \mathbb{I}}\left\langle f, g_{i}\right\rangle f_{i}, \quad \text { for } f \in \mathcal{W} .
$$

The representation above lies within the theory of oblique duality (see [17, 18, 22, 23]). In applied situations, it is usually desired to develop encoding-decoding schemes as above, with some additional features. In some cases, we search for schemes with prescribed properties (e.g., for which the sequence of norms $\left\{\left\|f_{i}\right\|^{2}\right\}_{i \in \mathbb{I}}$ as well as the spectral properties of the family $\mathcal{F}$ are given in advance) leading to what is known in the literature as frame design problem (see [3, 6, 12, 14, 35, 42]). In other cases, we search for numerically robust oblique dual pairs $(\mathcal{F}, \mathcal{G})$ as above, leading to what is known as optimal frame designs ([5, 18, 24, 36, 40, 47]).

In their seminal work [6], Benedetto and Fickus introduced a functional defined on finite sequences of (unit norm) vectors $\mathcal{F}=\left\{f_{i}\right\}_{i \in \mathbb{I}_{n}}$ (where $\mathbb{I}_{n}=\{1, \ldots, n\}$ ), the so-called frame potential, given by

$$
\mathrm{FP}(\mathcal{F})=\sum_{i, j \in \mathbb{I}_{n}}\left|\left\langle f_{i}, f_{j}\right\rangle\right|^{2}
$$

In case $\operatorname{dim} \mathcal{H}=d \in \mathbb{N}$ then one of their major results shows that tight unit norm frames can be characterized as (local) minimizers of this functional, among unit norm frames. Since then, there has been interest in (local) minimizers of the frame potential within certain classes of frames, since such minimizers can be considered as natural substitutes of tight frames (see for example [13, 36]). Recently, there has been interest in the structure of minimizers of other potentials such as the so-called mean squared error (MSE) (see [24, 37, 42] and the references therein). Both the frame potential and the MSE are examples of the so-called convex potentials introduced in [36]. It 
turns out that minimizers of these convex potentials share the spectral and geometrical structure of minimizers of the frame potential. Now, it is a well known fact that in case $\mathcal{V}=\mathcal{W}$ then tight frames $\mathcal{F}$ for $\mathcal{W}$ - i.e. minimizers of convex potentials - give rise to optimal (numerically robust) dual pairs $(\mathcal{F}, \mathcal{G})$. Therefore, it seems apparent that in the general case $\mathcal{V} \oplus \mathcal{W}^{\perp}$ the construction of robust oblique dual pairs $(\mathcal{F}, \mathcal{G})$ is related with the construction of frames $\mathcal{F}$ which are minimizers of convex potentials (e.g. the frame potential).

It turns out that there is a single notion that ties all the previous problems together namely, the majorization preorder. Indeed, majorization is the key notion behind the frame design problems (see [3, 12, 14]) through natural extensions of the Schur-Horn theorem from matrix analysis (see [3, 9, 10, 31]). Moreover, the relation between majorization and tracial inequalities with respect to convex functions allows to apply this notion in the study of convex potentials ([5, 36, 37, 38, 40]). Unfortunately, the convex potentials considered in 36. (in particular, the frame potential) can only be defined for finite frames. Hence, in the infinite dimensional context we loose a tool which have proved useful as a measure of stability for frames in finite dimensional Hilbert spaces.

In this paper we show that there are natural analogues of the convex potentials (and in particular, of the frame potential) in the context of Bessel sequences of integer translates $E(\mathcal{F})$ of a finite family of vectors $\mathcal{F}=\left\{f_{i}\right\}_{i \in \mathbb{I}_{n}}$ that lie in a finitely generated shift invariant (FSI) subspace $\mathcal{W}$ of $L^{2}\left(\mathbb{R}^{k}\right)$. We take advantage of the detailed structure of $E(\mathcal{F})$ as a fibered family over the torus $\mathbb{T}^{k}$ (see [7, 8, 43]) and the theory of range functions for shift invariant (SI) subspaces and introduce the potential $P_{\varphi}^{\mathcal{W}}(E(\mathcal{F}))$ associated to a convex function $\varphi$ and $\mathcal{W}$ as an integral over $\mathbb{T}^{k}$ of the corresponding potentials on the fibers (for related approaches to different problems in SI subspaces see [1, 28, 29]). In order to verify that our definition is a natural extension of the convex potentials for finite frames we show that under natural normalization conditions, a family of integer translates $E(\mathcal{F})$ that is a tight frame for a FSI subspace $\mathcal{W}$ is a minimizer of the convex potential associated to $\varphi$ and $\mathcal{W}$.

The convex potentials in FSI subspaces raise several questions related with optimal design problems. In particular, given FSI subspaces $\mathcal{W}, \mathcal{V}$ such that $\mathcal{V} \oplus \mathcal{W}^{\perp}=L^{2}\left(\mathbb{R}^{k}\right)$ and a finite family $\mathcal{F}=\left\{f_{i}\right\}_{i \in \mathbb{I}_{n}}$ such that $E(\mathcal{F})$ is a frame for $\mathcal{W}$, we consider the problem of designing optimal oblique duals $E(\mathcal{G})$ which are translates of a family $\mathcal{G}=\left\{g_{i}\right\}_{i \in \mathbb{I}_{n}}$ in $\mathcal{V}$ and such that $\mathcal{G}$ satisfies the norm restrictions $\sum_{i \in \mathbb{I}_{n}}\left\|g_{i}\right\|^{2} \geq w$, for $w>0$. In order to deal with this problem we develop two new tools in the context of frames of translates. On the one hand, we obtain what we call the fine spectral structure of shift generated $(\mathrm{SG})$ oblique dual frames of the fixed frame $E(\mathcal{F})$, which is a detailed description of the eigenvalues of the measurable field of positive operators defined on $\mathbb{T}^{k}$ corresponding to the frame operators of SG oblique duals of $E(\mathcal{F})$. As a consequence, we derive necessary and sufficient conditions for the existence of a tight SG oblique dual $E(\mathcal{G})$ of $E(\mathcal{F})$. On the other hand, we consider the water-filling construction (both for functions in probability spaces as well as for measurable field of positive finite-rank operators) and show that this construction leads to optimal solutions of the oblique dual design problem; this is achieved by showing that the water-filling constructions are optimal with respect to majorization (considered in the general context of probability spaces) which is a result of independent interest. With these tools we completely solve the problem of designing optimal oblique dual frames with norm restriction mentioned before; it turns out that these optimal SG oblique duals are more stable than the so-called canonical oblique dual. We point out that the structure of the optimal solution is obtained in terms of a global analysis. As a byproduct we extend the so-called Fan-Pall interlacing theorem from matrix analysis to the context of measurable fields of positive matrices.

The paper is organized as follows. In section 2 we describe several preliminary notions and facts from frame theory, SI subspaces, frames of translates and majorization theory in probability spaces. In section 3 we introduce the convex potentials for frames of translates and show that are natural extensions of the convex potentials for finite frames. In section 4 we recall several facts from the 
theory of oblique duality in FSI subspaces and obtain the precise value of the aliasing norm corresponding to the consistent sampling in this setting. Then, we describe the fine spectral structure of oblique duals of a fixed SG frame. Since this result depends on an extension of the Fan-Pall interlacing theory, its proof is presented in an appendix (see section 6). In section 5 we study the problem of optimal design of oblique dual frames $E(\mathcal{G})$ - of a fixed finitely SG frame $E(\mathcal{F})$ - which satisfy certain norm restrictions. We first show that the water-filling construction for positive functions in probability spaces is optimal with respect to sub-majorization within a natural set of functions. We then construct optimal SG oblique duals with norm restrictions and explain the relation of our construction with a natural (non-commutative) water-filling construction for measurable field of positive finite-rank operators. The paper ends with an appendix section in which we develop the Fan-Pall interlacing theorem for measurable fields of positive matrices as well as some consequences of this result.

\section{Preliminaries}

In this section we recall some basic facts related with frame theory, oblique duality and shift invariant (SI) subspaces of $L^{2}\left(\mathbb{R}^{k}\right)$. At the end of this section we describe majorization between functions in arbitrary probability spaces.

\section{General Notation}

Throughout this work we shall use the following notation: the space of complex $d \times d$ matrices is denoted by $\mathcal{M}_{d}(\mathbb{C})$ and $\mathcal{M}_{d}(\mathbb{C})^{+}$denotes the set of positive semidefinite matrices. $\mathcal{G l}(d)$ is the group of invertible elements of $\mathcal{M}_{d}(\mathbb{C})$ and $\mathcal{G l}(d)^{+}=\mathcal{M}_{d}(\mathbb{C})^{+} \cap \mathcal{G l}(d)$. If $T \in \mathcal{M}_{d}(\mathbb{C})$, we denote by $\|T\|$ its spectral norm, by $\operatorname{rk} T=\operatorname{dim} R(T)$ the $\operatorname{rank}$ of $T$, and by $\operatorname{tr} T$ the trace of $T$.

Given $d \in \mathbb{N}$ we denote by $\mathbb{I}_{d}=\{1, \ldots, d\} \subseteq \mathbb{N}$. For a vector $x \in \mathbb{R}^{m}$ we denote by $x^{\downarrow}$ (resp. $x^{\uparrow}$ ) the rearrangement of $x$ in decreasing (resp. increasing) order. We denote by $\left(\mathbb{R}^{d}\right)^{\downarrow}=\left\{x \in \mathbb{R}^{d}: x=x^{\downarrow}\right\}$ the set of downwards ordered vectors, and $\left(\mathbb{R}^{d}\right)^{\uparrow}=\left\{x \in \mathbb{R}^{d}: x=x^{\uparrow}\right\}$.

Given $S \in \mathcal{M}_{d}(\mathbb{C})^{+}$, we write $\lambda(S)=\lambda^{\downarrow}(S)=\left(\lambda_{1}(S), \ldots, \lambda_{d}(S)\right) \in\left(\mathbb{R}^{d}\right)^{\downarrow}$ the vector of eigenvalues of $S$ - counting multiplicities - arranged in decreasing order. Similarly we denote by $\lambda^{\uparrow}(S) \in\left(\mathbb{R}^{d}\right)^{\uparrow}$ the reverse ordered vector of eigenvalues of $S$.

If $W \subseteq \mathbb{C}^{d}$ is a subspace we denote by $P_{W} \in \mathcal{M}_{d}(\mathbb{C})^{+}$the orthogonal projection onto $W$. Given $x, y \in \mathbb{C}^{d}$ we denote by $x \otimes y \in \mathcal{M}_{d}(\mathbb{C})$ the rank one matrix given by

$$
x \otimes y(z)=\langle z, y\rangle x \quad \text { for every } \quad z \in \mathbb{C}^{d} .
$$

Note that, if $x \neq 0$, then the projection $P_{x} \stackrel{\text { def }}{=} P_{\text {span }\{x\}}=\|x\|^{-2} x \otimes x$.

\subsection{Frames for subspaces and oblique duality}

In what follows $\mathcal{H}$ denotes a separable complex Hilbert space and $\mathbb{I}$ denotes a finite or countable infinite set. Let $\mathcal{W}$ be a closed subspace of $\mathcal{H}$ : recall that a sequence $\mathcal{F}=\left\{f_{i}\right\}_{i \in \mathbb{I}}$ in $\mathcal{W}$ is a frame for $\mathcal{W}$ if there exist positive constants $0<a \leq b$ such that

$$
a\|f\|^{2} \leq \sum_{i \in \mathbb{I}}\left|\left\langle f, f_{i}\right\rangle\right|^{2} \leq b\|f\|^{2} \quad \text { for every } \quad f \in \mathcal{W} .
$$

In general, if $\mathcal{F}$ satisfies the inequality to the right in Eq. (3) we say that $\mathcal{F}$ is a $b$-Bessel sequence.

Given a Bessel sequence $\mathcal{F}=\left\{f_{i}\right\}_{i \in \mathbb{I}}$ we consider its synthesis operator $T_{\mathcal{F}} \in L\left(\ell^{2}(\mathbb{I}), \mathcal{H}\right)$ given by $T_{\mathcal{F}}\left(\left(a_{i}\right)_{i \in \mathbb{I}}\right)=\sum_{i \in \mathbb{I}} a_{i} f_{i}$ which, by hypothesis on $\mathcal{F}$, is a bounded linear transformation. We also 
consider $T_{\mathcal{F}}^{*} \in L\left(\mathcal{H}, \ell^{2}(\mathbb{I})\right)$ called the analysis operator of $\mathcal{F}$, given by $T_{\mathcal{F}}^{*}(f)=\left(\left\langle f, f_{i}\right\rangle\right)_{i \in \mathbb{I}}$ and the frame operator of $\mathcal{F}$ defined by $S_{\mathcal{F}}=T_{\mathcal{F}} T_{\mathcal{F}}^{*}$. It is straightforward to check that

$$
\left\langle S_{\mathcal{F}} f, f\right\rangle=\sum_{i \in \mathbb{I}}\left|\left\langle f, f_{i}\right\rangle\right|^{2} \quad \text { for every } \quad f \in \mathcal{H} .
$$

Hence, $S_{\mathcal{F}}$ is a positive semidefinite bounded operator; moreover, a Bessel sequence $\mathcal{F}$ in $\mathcal{W}$ is a frame for $\mathcal{W}$ if and only if $S_{\mathcal{F}}$ is an invertible operator when restricted to $\mathcal{W}$ or equivalently, if the range of $T_{\mathcal{F}}$ coincides with $\mathcal{W}$.

In order to describe oblique duality, we fix two closed subspaces $\mathcal{V}, \mathcal{W} \subseteq \mathcal{H}$ such that $\mathcal{W}^{\perp} \oplus \mathcal{V}=\mathcal{H}$, that is such that $\mathcal{W}^{\perp}+\mathcal{V}=\mathcal{H}$ and $\mathcal{W}^{\perp} \cap \mathcal{V}=\{0\}$. Hence, $\mathcal{W}^{\perp}$ is a common (algebraic) complement of $\mathcal{W}$ and $\mathcal{V}$. It is well known that in this case $P_{\mathcal{W}} \mid \mathcal{V}: \mathcal{V} \rightarrow \mathcal{W}$ is a linear bounded isomorphism so, in particular, we see that $\operatorname{dim} \mathcal{V}=\operatorname{dim} \mathcal{W}$ as Hilbert spaces. Moreover, the conditions $\mathcal{W}^{\perp} \oplus \mathcal{V}=\mathcal{H}$ and $\mathcal{W} \oplus \mathcal{V}^{\perp}=\mathcal{H}$ are actually equivalent.

Fix a frame $\mathcal{F}=\left\{f_{i}\right\}_{i \in \mathbb{I}}$ for $\mathcal{W}$. Following [22, 23] (see also [18]), given a Bessel sequence $\mathcal{G}=\left\{g_{i}\right\}_{i \in \mathbb{I}}$ in $\mathcal{V}$ we say that $\mathcal{G}$ is a (oblique) $\mathcal{V}$-dual of $\mathcal{F}$ if

$$
f=\sum_{i \in \mathbb{I}}\left\langle f, g_{i}\right\rangle f_{i} \quad \text { for every } \quad f \in \mathcal{W} .
$$

It turns out (see [22, 23]) that if $\mathcal{G}$ is a $\mathcal{V}$-dual of $\mathcal{F}$ then $T_{\mathcal{F}} T_{\mathcal{G}}^{*}=P_{\mathcal{W} / \mathcal{V}^{\perp}}$, where $P_{\mathcal{W} / \mathcal{V}^{\perp}}$ denotes the oblique projection with range $\mathcal{W}$ and null space $\mathcal{V}^{\perp}$. In this case, by taking adjoints in the

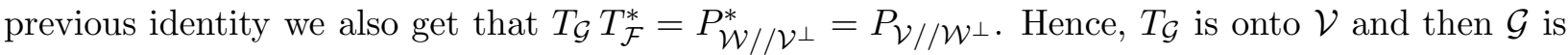
a frame for $\mathcal{V}$; moreover, we obtain the reconstruction formula

$$
g=\sum_{i \in \mathbb{I}}\left\langle g, f_{i}\right\rangle g_{i} \quad \text { for every } \quad g \in \mathcal{V} .
$$

We consider the set of oblique $\mathcal{V}$-duals of $\mathcal{F}$ given by

$$
\mathcal{D} \mathcal{V}(\mathcal{F})=\left\{\mathcal{G}=\left\{g_{i}\right\}_{i \in \mathbb{I}} \text { is a } \mathcal{V} \text {-dual of } \mathcal{F}\right\} .
$$

Remark 2.1. Let $\mathcal{F}=\left\{f_{i}\right\}_{i \in \mathbb{I}}$ be a frame for $\mathcal{W}$. If we set $\mathcal{V}=\mathcal{W}$ then a Bessel sequence $\mathcal{G}$ in $\mathcal{W}$ is a $\mathcal{W}$-dual of $\mathcal{F}$ if it is a dual frame for $\mathcal{F}$ in $\mathcal{W}$ in the classical sense (see [16]) i.e. $T_{\mathcal{G}} T_{\mathcal{F}}^{*}=P_{\mathcal{W}}$. Hence

$$
\mathcal{D}_{\mathcal{W}}(\mathcal{F})=\mathcal{D}(\mathcal{F}) \stackrel{\text { def }}{=}\left\{\mathcal{G}=\left\{g_{i}\right\}_{i \in \mathbb{I}} \text { is a dual frame for } \mathcal{F} \text { in } \mathcal{W}\right\} \text {. }
$$

Recall that there is a distinguished (classical) dual, called the canonical dual of $\mathcal{F}$, denoted $\mathcal{F}^{\#}=$ $\left\{f_{i}^{\#}\right\}_{i \in \mathbb{I}}$, given by $f_{i}^{\#}=S_{\mathcal{F}}^{\dagger} f_{i}$ for $i \in \mathbb{I}$, where $S_{\mathcal{F}}^{\dagger}$ denotes the Moore-Penrose pseudo-inverse of the (closed range positive semidefinite operator ) $S_{\mathcal{F}}$.

In the general context of oblique duality there also exists a distinguished $\mathcal{V}$-dual for $\mathcal{F}$, the so-called canonical $\mathcal{V}$-dual, which we denote by

$$
\mathcal{F}_{\mathcal{V}}^{\#}=\left\{f_{\mathcal{V}, i}^{\#}\right\}_{i \in \mathbb{I}} \quad \text { given by } \quad f_{\mathcal{V}, i}^{\#}=P_{\mathcal{V} / / \mathcal{W}^{\perp}} f_{i}^{\#} \quad \text { for every } \quad i \in \mathbb{I},
$$

where $\mathcal{F}^{\#}=\left\{f_{i}^{\#}\right\}_{i \in \mathbb{I}}$ is the canonical dual of $\mathcal{F}$. It turns out that the encoding-decoding scheme based on the oblique dual pair $\left(\mathcal{F}, \mathcal{F}_{\mathcal{V}}^{\#}\right)$ has several optimality properties (see [22, 23]). 


\subsection{Shift-invariant subspaces and frames of translates}

In what follows we consider $L^{2}\left(\mathbb{R}^{k}\right)$ (with respect to Lebesgue measure) as a separable and complex Hilbert space. Recall that a closed subspace $\mathcal{V} \subseteq L^{2}\left(\mathbb{R}^{k}\right)$ is shift-invariant (SI) if $f \in \mathcal{V}$ implies $T_{\ell} f \in \mathcal{V}$ for any $\ell \in \mathbb{Z}^{k}$, where $T_{y} f(x)=f(x-y)$ is the translation by $y \in \mathbb{R}^{k}$. For example, if we take a subset $\mathcal{A} \subset L^{2}\left(\mathbb{R}^{k}\right)$ then,

$$
\mathcal{S}(\mathcal{A})=\overline{\operatorname{span}}\left\{T_{\ell} f: f \in \mathcal{A}, \ell \in \mathbb{Z}^{k}\right\}
$$

is a shift-invariant subspace called the $S I$ subspace generated by $\mathcal{A}$. Indeed, $\mathcal{S}(\mathcal{A})$ is the smallest SI subspace that contains $\mathcal{A}$. We say that a SI subspace $\mathcal{V}$ is finitely generated (FSI) if there exists a finite set $\mathcal{A} \subset L^{2}\left(\mathbb{R}^{k}\right)$ such that $\mathcal{V}=S(\mathcal{A})$. In this case, the length of $\mathcal{V}$ is the smallest cardinal $\#(\mathcal{A})$ such that $S(\mathcal{A})=\mathcal{V}$.

In order to describe the fine structure of a SI subspace we consider the following representation of $L^{2}\left(\mathbb{R}^{k}\right)$ (see [7, 8, 27, 43] and [11] for extensions of these notions to the more general context of actions of locally compact abelian groups). Let $\mathbb{T}=[-1 / 2,1 / 2)$ be endowed with the Lebesgue measure and let $L^{2}\left(\mathbb{T}^{k}, \ell^{2}\left(\mathbb{Z}^{k}\right)\right)$ be the Hilbert space of square integrable $\ell^{2}\left(\mathbb{Z}^{k}\right)$-valued functions that consists of all vector valued measurable functions $\phi: \mathbb{T}^{k} \rightarrow \ell^{2}\left(\mathbb{Z}^{k}\right)$ with the norm

$$
\|\phi\|^{2}=\int_{\mathbb{T}^{k}}\|\phi(x)\|_{\ell^{2}\left(\mathbb{Z}^{k}\right)}^{2} d x<\infty .
$$

Then, $\Gamma: L^{2}\left(\mathbb{R}^{k}\right) \rightarrow L^{2}\left(\mathbb{T}^{k}, \ell^{2}\left(\mathbb{Z}^{k}\right)\right)$ defined for $f \in L^{1}\left(\mathbb{R}^{k}\right) \cap L^{2}\left(\mathbb{R}^{k}\right)$ by

$$
\Gamma f: \mathbb{T}^{k} \rightarrow \ell^{2}\left(\mathbb{Z}^{k}\right), \quad \Gamma f(x)=(\hat{f}(x+\ell))_{\ell \in \mathbb{Z}^{k}},
$$

extends uniquely to an isometric isomorphism between $L^{2}\left(\mathbb{R}^{k}\right)$ and $L^{2}\left(\mathbb{T}^{k}, \ell^{2}\left(\mathbb{Z}^{k}\right)\right)$; here $\hat{f}$ denotes the Fourier transform of $f \in L^{2}\left(\mathbb{R}^{k}\right)$.

Let $\mathcal{V} \subset L^{2}\left(\mathbb{R}^{k}\right)$ be a SI subspace. Then, there exists a function $J_{\mathcal{V}}: \mathbb{T}^{k} \rightarrow\{$ closed subspaces of $\left.\ell^{2}\left(\mathbb{Z}^{k}\right)\right\}$ such that: if $P_{J_{\mathcal{V}}(x)}$ denotes the orthogonal projection onto $J_{\mathcal{V}}(x)$ for $x \in \mathbb{T}^{k}$, then for every $\xi, \eta \in \ell^{2}\left(\mathbb{Z}^{k}\right)$ the function $x \mapsto\left\langle P_{J_{\mathcal{V}}(x)} \xi, \eta\right\rangle$ is measurable and

$$
\mathcal{V}=\left\{f \in L^{2}\left(\mathbb{R}^{k}\right): \Gamma f(x) \in J_{\mathcal{V}}(x) \text { for a.e. } x \in \mathbb{T}^{k}\right\} .
$$

The funcion $J_{\mathcal{V}}$ is the so-called measurable range function associated with $\mathcal{V}$. By [8, Prop.1.5], Eq. (7) establishes a bijection between SI subspaces of $L^{2}\left(\mathbb{R}^{k}\right)$ and measurable range functions. In case $\mathcal{V}=S(\mathcal{A}) \subseteq L^{2}\left(\mathbb{R}^{k}\right)$ is the SI subspace generated by $\mathcal{A}=\left\{h_{i}: i \in \mathbb{I}\right\} \subset L^{2}\left(\mathbb{R}^{k}\right)$, where $\mathbb{I}$ is a finite or countable infinite set, then for a.e. $x \in \mathbb{T}^{k}$ we have that

$$
J_{\mathcal{V}}(x)=\left\{\Gamma h_{i}(x): i \in \mathbb{I}\right\}^{-\|\cdot\|} .
$$

Recall that a bounded linear transformation $S \in L\left(L^{2}\left(\mathbb{R}^{k}\right)\right)$ is shift preserving (SP) if $T_{\ell} S=S T_{\ell}$ for every $\ell \in \mathbb{Z}^{k}$. In this case (see [8, Thm 4.5]) there exists a (weakly) measurable field of operators $[S]_{(\cdot)}: \mathbb{T}^{k} \rightarrow \ell^{2}\left(\mathbb{Z}^{k}\right)$ (i.e. such that for every $\xi, \eta \in \ell^{2}\left(\mathbb{Z}^{k}\right)$ the function $\mathbb{T}^{k} \ni x \mapsto\left\langle[S]_{x} \xi, \eta\right\rangle$ is measurable) and essentially bounded (i.e. the function $\mathbb{T}^{k} \ni x \mapsto\left\|[S]_{x}\right\|$ is essentially bounded) such that

$$
[S]_{x}(\Gamma f(x))=\Gamma(S f)(x) \quad \text { for a.e. } x \in \mathbb{T}^{k}, \quad f \in L^{2}\left(\mathbb{R}^{k}\right) .
$$

Moreover, $\|S\|=\operatorname{ess}_{\sup } \in \mathbb{T}^{k}\left\|[S]_{x}\right\|$. Conversely, if $s: \mathbb{T}^{k} \rightarrow L\left(\ell^{2}\left(\mathbb{Z}^{k}\right)\right)$ is a weakly measurable and essentially bounded field of operators then, there exists a unique bounded operator $S \in L\left(L^{2}\left(\mathbb{R}^{k}\right)\right)$ that is $\mathrm{SP}$ and such that $[S]=s$. For example, let $\mathcal{V}$ be a SI subspace and consider $P \mathcal{V} \in L\left(L^{2}\left(\mathbb{R}^{k}\right)\right)$, the orthogonal projection onto $\mathcal{V}$; then, $P_{\mathcal{V}}$ is SP so that $\left[P_{\mathcal{V}}\right]: \mathbb{T}^{k} \rightarrow L\left(\ell^{2}\left(\mathbb{Z}^{k}\right)\right)$ is given by $\left[P_{\mathcal{V}}\right]_{x}=P_{J_{\mathcal{V}}(x)}$ i.e., the orthogonal projection onto $J_{\mathcal{V}}(x)$, for a.e. $x \in \mathbb{T}^{k}$. 
The previous notions associated with SI subspaces and SP operators allow to develop a detailed study of frames of translates. Indeed, let $\mathcal{F}=\left\{f_{i}\right\}_{i \in \mathbb{I}}$ be a (possibly finite) sequence in $L^{2}\left(\mathbb{R}^{k}\right)$. We denote by $E(\mathcal{F})$ the family of translates of $\mathcal{F}$, namely $E(\mathcal{F})=\left\{T_{\ell} f_{i}\right\}_{(\ell, i) \in \mathbb{Z}^{k} \times \mathbb{I}}$. For $x \in \mathbb{T}^{k}$, let $\Gamma \mathcal{F}(x)=\left\{\Gamma f_{i}(x)\right\}_{i \in \mathbb{I}}$ which is a (possibly finite) sequence in $\ell^{2}\left(\mathbb{Z}^{k}\right)$. Then [8, 43] $E(\mathcal{F})$ is a $b$-Bessel sequence if and only if $\Gamma \mathcal{F}(x)$ is a $b$-Bessel sequence for a.e. $x \in \mathbb{T}^{k}$. In this case, we consider $T_{\Gamma \mathcal{F}(x)}: \ell^{2}(\mathbb{I}) \rightarrow \ell^{2}\left(\mathbb{Z}^{k}\right)$ and $S_{\Gamma \mathcal{F}(x)}: \ell^{2}\left(\mathbb{Z}^{k}\right) \rightarrow \ell^{2}\left(\mathbb{Z}^{k}\right)$ the synthesis and frame operators of $\Gamma \mathcal{F}(x)$, respectively, for $x \in \mathbb{T}^{k}$; it is straightforward to check that $S_{E(\mathcal{F})}$ is a SP operator.

If $\mathcal{F}=\left\{f_{i}\right\}_{i \in \mathbb{I}}$ and $\mathcal{G}=\left\{g_{i}\right\}_{i \in \mathbb{I}}$ are such that $E(\mathcal{F})$ and $E(\mathcal{G})$ are Bessel sequences then (see [28, 43]) the following fundamental relation holds:

$$
\left[T_{E(\mathcal{G})} T_{E(\mathcal{F})}^{*}\right]_{x}=T_{\Gamma \mathcal{G}(x)} T_{\Gamma \mathcal{F}(x)}^{*}, \quad \text { for a.e } x \in \mathbb{T}^{k} .
$$

Eq. (10) has several consequences; indeed, if $\mathcal{W}$ is a SI subspace of $L^{2}\left(\mathbb{R}^{k}\right)$ and we assume further that $\mathcal{F}, \mathcal{G} \in \mathcal{W}^{n}$ then:

1. For every $f, g \in L^{2}\left(\mathbb{R}^{k}\right)$,

$$
\left\langle S_{E(\mathcal{F})} f, g\right\rangle=\int_{\mathbb{T}^{k}}\left\langle S_{\Gamma \mathcal{F}(x)} \Gamma f(x), \Gamma g(x)\right\rangle_{\ell^{2}\left(\mathbb{Z}^{k}\right)} d x .
$$

This last fact implies that $\left[S_{E(\mathcal{F})}\right]_{x}=S_{\Gamma \mathcal{F}(x)}$ for a.e. $x \in \mathbb{T}^{k}$; moreover, it also holds that $E(\mathcal{F})$ is a frame for $\mathcal{W}$ with frame bounds $0<a \leq b$ if and only if $\Gamma \mathcal{F}(x)$ is a frame for $J_{\mathcal{W}}(x)$ with frame bounds $0<a \leq b$ for a.e. $x \in \mathbb{T}^{k}$ (see [8]).

2. Since $\left[P_{\mathcal{W}}\right]_{x}=P_{J_{\mathcal{W}}(x)}$ for a.e. $x \in \mathbb{T}^{k}$ then $E(\mathcal{G})$ is a (classical) dual for $E(\mathcal{F})$ in $\mathcal{W}$ if and only if $\Gamma \mathcal{G}(x)$ is a (classical) dual for $\Gamma \mathcal{F}(x)$ in $J_{\mathcal{W}}(x)$ for a.e. $x \in \mathbb{T}^{k}$ (see [8, 28, 29]).

\subsection{Majorization in probability spaces}

Majorization between vectors (see [4, 34]) has played a key role in frame theory. On the one hand, majorization allows to characterize the existence of frames with prescribed properties (see [3, 12, 14]). On the other hand, majorization is a preorder relation that implies a family of tracial inequalities; this last fact can be used to explain the structure of minimizers of the so-called Benedetto-Fickus frame potential $([6,13)$ as well as more general convex potentials for finite frames (see [35, 36, 37, 38, 40]). In the next section we extend the notion of convex potentials to the context of Bessel families of translates of finite sequences; therefore, we will need the following general notion of majorization between functions in probability spaces.

Throughout this section the triple $(X, \mathcal{X}, \mu)$ denotes a probability space i.e. $\mathcal{X}$ is a $\sigma$-algebra of sets in $X$ and $\mu$ is a probability measure defined on $\mathcal{X}$. We shall denote by $L^{\infty}(X, \mu)^{+}=\{f \in$ $\left.L^{\infty}(X, \mu): f \geq 0\right\}$. For $f \in L^{\infty}(X, \mu)^{+}$, the decreasing rearrangement of $f$ (see [34]), denoted $f^{*}:[0,1) \rightarrow \mathbb{R}^{+}$, is given by

$$
f^{*}(s) \stackrel{\text { def }}{=} \sup \left\{t \in \mathbb{R}^{+}: \mu\{x \in X: f(x)>t\}>s\right\} \quad \text { for every } \quad s \in[0,1) .
$$

Remark 2.2. We mention some elementary facts related with the decreasing rearrangement of functions that we shall need in the sequel. Let $f \in L^{\infty}(X, \mu)^{+}$, then:

1. $f^{*}$ is a right-continuous and non-increasing function.

2. $f$ and $f^{*}$ are equimeasurable i.e. for every Borel set $A \subset \mathbb{R}$ then $\mu\left(f^{-1}(A)\right)=\left|\left(f^{*}\right)^{-1}(A)\right|$, where $|B|$ denotes the Lebesgue measure of the Lebesgue measurable set $B \subset \mathbb{R}$. In turn, this implies that for every continuous $\varphi: \mathbb{R}^{+} \rightarrow \mathbb{R}^{+}$then: $\varphi \circ f \in L^{\infty}(X, \mu)$ iff $\varphi \circ f^{*} \in L^{\infty}([0,1])$ and in this case

$$
\int_{X} \varphi \circ f d \mu=\int_{0}^{1} \varphi \circ f^{*} d x
$$


3. If $g \in L^{\infty}(X, \mu)$ is such that $f \leq g$ then $0 \leq f^{*} \leq g^{*}$; moreover, in case $f^{*}=g^{*}$ then $f=g$.

4. If we consider the probability space $([0,1], \mathcal{B}, d t)$ - Lebesgue measurable sets in $[0,1]$ with Lebesgue measure - then $f^{*} \in L^{\infty}([0,1], d t)$ is such that $\left(f^{*}\right)^{*}=f^{*}$.

5. If $c \in \mathbb{R}$ is such that $f+c \geq 0$ then $(f+c)^{*}=f^{*}+c$.

Definition 2.3. Let $f, g \in L^{\infty}(X, \mu)^{+}$and let $f^{*}, g^{*}$ denote their decreasing rearrangements. We say that $f$ submajorizes $g$ (in $(X, \mathcal{X}, \mu)$ ), denoted $g \prec{ }_{w} f$, if

$$
\int_{0}^{s} g^{*}(t) d t \leq \int_{0}^{s} f^{*}(t) d t \quad \text { for every } \quad 0 \leq s \leq 1 .
$$

If we further have that $\int_{0}^{1} g^{*}(t) d t=\int_{0}^{1} f^{*}(t) d t$ we say that $f$ majorizes $g$ and write $g \prec f$.

In order to check majorization between functions in probability spaces, we can consider the so-called doubly stochastic maps. Recall that a linear operator $D$ acting on $L^{\infty}(X, \mu)$ is a doubly-stochastic map if $D$ is unital, positive and trace preserving i.e.

$$
D\left(1_{X}\right)=1_{X}, \quad D\left(L^{\infty}(X, \mu)^{+}\right) \subseteq L^{\infty}(X, \mu)^{+} \quad \text { and } \quad \int_{X} D(f)(x) d \mu(x)=\int_{X} f(x) d \mu(x)
$$

for every $f \in L^{\infty}(X, \mu)$. It is worth pointing out that $D$ is necessarily a contractive map.

Our interest in majorization relies in its relation with integral inequalities in terms of convex functions. The following result summarizes this relation as well as the role of the doubly stochastic maps (see for example [15, 44]).

Theorem 2.4. Let $f, g \in L^{\infty}(X, \mu)^{+}$. Then the following conditions are equivalent:

1. $g \prec f$;

2. There is a doubly stochastic map $D$ acting on $L^{\infty}(X, \mu)$ such that $D(f)=g$;

3. For every convex function $\varphi: \mathbb{R}^{+} \rightarrow \mathbb{R}^{+}$we have that

$$
\int_{X} \varphi(g(x)) d \mu(x) \leq \int_{X} \varphi(f(x)) d \mu(x) .
$$

In case we only have $g \prec_{w} f$ then Eq. (12) holds if we assume further that $\varphi$ is an increasing convex function.

Example 2.5. The operator $D$ given by $D(f)=\left(\int_{X} f d \mu\right) \cdot 1_{X}$ is a doubly stochastic map. Hence, we get the majorization relation $\left(\int_{X} f d \mu\right) \cdot 1_{X} \prec f$. Therefore, if $\varphi: \mathbb{R}^{+} \rightarrow \mathbb{R}^{+}$is any convex function and $f \in L^{\infty}(X, \mu)^{+}$then, by Theorem 2.4 , we have that

$$
\varphi\left(\int_{X} f d \mu\right)=\int_{X} \varphi\left(\left(\int_{X} f d \mu\right) \cdot 1_{X}(x)\right) d \mu(x) \leq \int_{X} \varphi(f(x)) d \mu(x),
$$

which is an instance of the classical Jensen's inequality. Using the previous facts, notice that if $c \in \mathbb{R}$ is such that $0 \leq c \leq \int_{X} f d \mu$ then it is easy to see that $c \cdot 1_{X} \prec{ }_{w} f$.

The following result will play a key role in the study of the structure of minimizers of $\prec_{w}$ within (appropriate) sets of functions.

Proposition 2.6 ([15]). Let $f, g \in L^{\infty}(X, \mu)^{+}$such that $g \prec_{w} f$. If there exists a non-decreasing and strictly convex function $\varphi: \mathbb{R}^{+} \rightarrow \mathbb{R}^{+}$such that

$$
\int_{X} \varphi(f(x)) d \mu(x)=\int_{X} \varphi(g(x)) d \mu(x) \quad \text { then } \quad g^{*}=f^{*} .
$$

With the notations of Example 2.5 notice that Proposition 2.6 implies (the known fact) that if $\varphi$ is strictly convex and such that equality holds in Jensen's inequality Eq. (13) then $f^{*}=\int_{X} f d \mu$ and hence $f=\int_{X} f d \mu$. 


\section{Convex potentials for sequences of translates in FSI spaces}

We begin by describing the convex potentials for finite sequences of vectors with respect to a finite dimensional subspace. We consider the sets

$$
\operatorname{Conv}\left(\mathbb{R}^{+}\right)=\left\{\varphi: \mathbb{R}^{+} \rightarrow \mathbb{R}^{+}, \varphi \text { is a convex function }\right\}
$$

and $\operatorname{Conv}_{\mathrm{s}}\left(\mathbb{R}^{+}\right)=\left\{\varphi \in \operatorname{Conv}\left(\mathbb{R}^{+}\right), \varphi\right.$ is strictly convex $\}$.

Now, given $\varphi \in \operatorname{Conv}\left(\mathbb{R}^{+}\right)$and a finite dimensional subspace $\mathcal{W} \subset \mathcal{H}$, then the convex potential associated to $(\varphi, \mathcal{W})$, denoted by $P_{\varphi}^{\mathcal{W}}$, is defined as follows: for a finite sequence $\mathcal{F}=\left\{f_{i}\right\}_{i \in \mathbb{I}_{n}} \in \mathcal{W}^{n}$ with frame operator $S_{\mathcal{F}} \in L(\mathcal{H})^{+}$,

$$
P_{\varphi}^{\mathcal{W}}(\mathcal{F})=\operatorname{tr}\left[\varphi\left(S_{\mathcal{F}}\right) P_{\mathcal{W}}\right]
$$

where $\varphi\left(S_{\mathcal{F}}\right) \in L(\mathcal{H})^{+}$is obtained by functional calculus and $\operatorname{tr}(\cdot)$ denotes the usual (semi-finite) trace in $L(\mathcal{H})$. Notice that by construction, $P_{\mathcal{W}} S_{\mathcal{F}}=S_{\mathcal{F}} P_{\mathcal{W}}=S_{\mathcal{F}}$ : then, it is clear that

$$
P_{\varphi}^{\mathcal{W}}(\mathcal{F})=\sum_{i \in \mathbb{I}_{d}} \varphi\left(\lambda_{i}\left(S_{\mathcal{F}}\right)\right)
$$

where $d=\operatorname{dim} \mathcal{W}$ and $\left(\lambda_{i}\left(S_{\mathcal{F}}\right)\right)_{i \in \mathbb{I}_{d}} \in\left(\mathbb{R}^{+}\right)^{d}$ denotes the vector of eigenvalues of the positive operator $\left.S_{\mathcal{F}}\right|_{\mathcal{W}} \in L(\mathcal{W})^{+}$, counting multiplicities and arranged in non-increasing order (we use the convention $\left.\mathbb{I}_{0}=\emptyset\right)$. In particular, if $\varphi \in \operatorname{Conv}\left(\mathbb{R}^{+}\right)$is such that $\varphi(0)=0$ we get that

$$
P_{\varphi}^{\mathcal{W}}(\mathcal{F})=\operatorname{tr}\left(\varphi\left(S_{\mathcal{F}}\right)\right)=\operatorname{tr}\left(\varphi\left(G_{\mathcal{F}}\right)\right)
$$

where the $n \times n$ matrix $G_{\mathcal{F}}=\left(\left\langle f_{i}, f_{j}\right\rangle\right)_{i, j \in \mathbb{I}_{n}}$ is the Gramian matrix of the finite sequence $\mathcal{F}$. That is, if $\varphi(0)=0$, then $P_{\varphi}^{\mathcal{W}}=P_{\varphi}$ does not depend on $\mathcal{W}$. For example, in case $\varphi(x)=x^{2}$, then $P_{\varphi}^{\mathcal{W}}(\mathcal{F})=P_{\varphi}(\mathcal{F})$ coincides with the frame potential: indeed, by Eq. (11) we have that

$$
P_{\varphi}^{\mathcal{W}}(\mathcal{F})=P_{\varphi}(\mathcal{F})=\operatorname{tr}\left(S_{\mathcal{F}}^{2}\right)=\operatorname{tr}\left(G_{\mathcal{F}}^{2}\right)=\sum_{i, j \in \mathbb{I}_{n}}\left|\left\langle f_{i}, f_{j}\right\rangle\right|^{2}=\mathrm{FP}(\mathcal{F})
$$

For $\varphi \in \operatorname{Conv}\left(\mathbb{R}^{+}\right)$and a finite dimensional subspace $\mathcal{W} \subset \mathcal{H}, P_{\varphi}^{\mathcal{W}}(\mathcal{F})$ is a measure of the spread of the eigenvalues of the frame operator of $\mathcal{F}=\left\{f_{i}\right\}_{i \in \mathbb{I}_{n}} \in \mathcal{W}^{n}$. That is, (under suitable normalization hypothesis on $\mathcal{F}$ ) the smaller the value $P_{\varphi}^{\mathcal{W}}(\mathcal{F})$ is, the more concentrated the eigenvalues of $\left.S_{\mathcal{F}}\right|_{\mathcal{W}} \in$ $L(\mathcal{W})^{+}$are. This is the main motivation for considering these convex potentials (see [36, 37, 40, 42]).

Next we extend the notion of convex potential to the context of finitely generated shift invariant systems in FSI subspaces.

Definition 3.1. Let $\mathcal{W}$ be a FSI subspace in $L^{2}\left(\mathbb{R}^{k}\right)$, let $\mathcal{F}=\left\{f_{i}\right\}_{i \in \mathbb{I}_{n}} \in \mathcal{W}^{n}$ be such that $E(\mathcal{F})$ is a Bessel sequence and consider $\varphi \in \operatorname{Conv}\left(\mathbb{R}^{+}\right)$. Then the convex potential associated to $(\varphi, \mathcal{W})$ on $E(\mathcal{F})$, denoted $P_{\varphi}^{\mathcal{W}}(E(\mathcal{F}))$, is given by

$$
P_{\varphi}^{\mathcal{W}}(E(\mathcal{F}))=\int_{\mathbb{T}^{k}} P_{\varphi}^{J_{\mathcal{W}}(x)}(\Gamma \mathcal{F}(x)) d x
$$

where $P_{\varphi}^{J_{\mathcal{W}}(x)}(\Gamma \mathcal{F}(x))=\operatorname{tr}\left(\varphi\left(S_{\Gamma \mathcal{F}(x)}\right)\left[P_{\mathcal{W}}\right]_{x}\right)$ is the convex potential associated with $\left(\varphi, J_{\mathcal{W}}(x)\right)$ of the sequence $\Gamma \mathcal{F}(x)=\left\{\Gamma f_{i}(x)\right\}_{i \in \mathbb{I}_{n}}$ in $\ell^{2}\left(\mathbb{Z}^{k}\right)$, for every $x \in \mathbb{T}^{k}$.

Next we develop some notions and tools in order to show that the right hand side in Eq. (17) is well defined, namely that the function $\mathbb{T}^{k} \ni x \mapsto P_{\varphi}^{J_{\mathcal{W}}(x)}(\Gamma \mathcal{F}(x))$ is integrable. 
Let $\mathcal{F}=\left\{f_{i}\right\}_{i \in \mathbb{I}_{n}}$ be a finite sequence in $L^{2}\left(\mathbb{R}^{k}\right)$ such that $E(\mathcal{F})$ is a Bessel sequence. Recall that in this case $S_{E(\mathcal{F})}$ is a SP operator and that for a.e. $x \in \mathbb{T}^{k},\left[S_{E(\mathcal{F})}\right]_{x}=S_{\Gamma \mathcal{F}(x)} \in L\left(\ell^{2}\left(\mathbb{Z}^{k}\right)\right)^{+}$is a positive and finite rank operator.

The next lemma is a reformulation of a result in [43] concerning the existence of measurable functions of eigenvalues and eigenvectors of measurable fields of positive semidefinite $n \times n$ matrices.

Lemma 3.2. Let $\mathcal{W}$ be a FSI subspace in $L^{2}\left(\mathbb{R}^{k}\right)$ and let $\mathcal{F}=\left\{f_{i}\right\}_{i \in \mathbb{I}_{n}} \in \mathcal{W}^{n}$ be such that $E(\mathcal{F})$ is a Bessel sequence. Then, there exist:

1. a measurable function $r: \mathbb{T}^{k} \rightarrow \mathbb{N}_{\geq 0}$ and measurable vector fields $v_{j}: \mathbb{T}^{k} \rightarrow \ell^{2}\left(\mathbb{Z}^{k}\right)$ for $j \in \mathbb{I}_{n}$ such that $r(x) \leq n$ and $\left\{v_{j}(x)\right\}_{j=1}^{r(x)}$ is an orthonormal system in $J_{\mathcal{W}}(x)$ for a.e. $x \in \mathbb{T}^{k}$;

2. bounded measurable functions $\lambda_{j}: \mathbb{T}^{k} \rightarrow \mathbb{R}^{+}$for $j \in \mathbb{I}_{n}$, such that $\lambda_{1} \geq \ldots \geq \lambda_{n}, \lambda_{j}(x)=0$ if $j>r(x)$ and

$$
\left[S_{E(\mathcal{F})}\right]_{x}=\sum_{j=1}^{r(x)} \lambda_{j}(x) v_{j}(x) \otimes v_{j}(x), \quad \text { for a.e. } x \in \mathbb{T}^{k}
$$

If we assume further that $E(\mathcal{F})$ is a frame for $\mathcal{W}$ then $r(x)=\operatorname{dim} J_{\mathcal{W}}(x)$ and $\left\{v_{j}(x)\right\}_{j=1}^{r(x)}$ is an orthonormal basis (ONB) for $J_{\mathcal{W}}(x)$ for a.e. $x \in \mathbb{T}^{k}$.

Proof. Consider the measurable field of positive semidefinite matrices $G: \mathbb{T}^{k} \rightarrow \mathcal{M}_{n}(\mathbb{C})^{+}$given by the Gramian $G(x)=\left(\left\langle\Gamma f_{i}(x), \Gamma f_{j}(x)\right\rangle\right)_{i, j \in \mathbb{I}_{n}}$, for $x \in \mathbb{T}^{k}$. Notice that $G(x)$ is the matrix representation of $T_{\Gamma \mathcal{F}(x)}^{*} T_{\Gamma \mathcal{F}(x)} \in L\left(\mathbb{C}^{n}\right)^{+}$with respect to the canonical basis of $\mathbb{C}^{n}$ for $x \in \mathbb{T}^{k}$. In particular, if $b$ denotes a Bessel (upper) bound of $E(\mathcal{F})$ then

$$
\operatorname{ess} \sup _{x \in \mathbb{T}^{k}}\|G(x)\|=\operatorname{ess} \sup _{x \in \mathbb{T}^{k}}\left\|T_{\Gamma \mathcal{F}(x)} T_{\Gamma \mathcal{F}(x)}^{*}\right\|=\left\|S_{E(\mathcal{F})}\right\| \leq b,
$$

by the remarks at the end of Section 2.2. We set $r(x)=\operatorname{rk}(G(x))=\operatorname{rk}\left(S_{\Gamma \mathcal{F}(x)}\right)$ for $x \in \mathbb{T}^{k}$; therefore $r(\cdot): \mathbb{T}^{k} \rightarrow \mathbb{N}_{\geq 0}$ is a measurable function such that $r(x) \leq n$ for $x \in \mathbb{T}^{k}$. Hence, by considering a convenient finite partition of $\mathbb{T}^{k}$ into measurable sets we can assume, without loss of generality, that $r(x)=r \in \mathbb{N}$ for a.e. $x \in \mathbb{T}^{k}$.

Using results from [43, we see that there exist measurable functions $\lambda_{j}: \mathbb{T}^{k} \rightarrow \mathbb{R}^{+}$and measurable vector fields $u_{j}: \mathbb{T}^{k} \rightarrow \mathbb{C}^{n}$, for $j \in \mathbb{I}_{n}$, such that: $\lambda_{j}(x) \geq \lambda_{j+1}(x)$ for $j \in \mathbb{I}_{n-1},\left\{u_{j}(x)\right\}_{j \in \mathbb{I}_{n}}$ is an ONB of $\mathbb{C}^{n}$ and $G(x) u_{j}(x)=\lambda_{j}(x) u_{j}(x)$ for $j \in \mathbb{I}_{n}$ and a.e. $x \in \mathbb{T}^{k}$. In particular, the functions $\lambda_{j}: \mathbb{T}^{k} \rightarrow \mathbb{R}^{+}$satisfy $0 \leq \lambda_{j}(x) \leq\|G(x)\| \leq b$ for a.e. $x \in \mathbb{T}^{k}, j \in \mathbb{I}_{n}$; these remarks prove item 2 above.

Take the polar decomposition $T_{\Gamma \mathcal{F}(x)}=U(x)\left|T_{\Gamma \mathcal{F}(x)}\right|$, where $U(x): \mathbb{C}^{n} \rightarrow J_{\mathcal{W}}(x) \subset \ell^{2}\left(\mathbb{Z}^{k}\right)$ is (the unique) partial isometry with $\operatorname{ker} U(x)=\operatorname{ker} T_{\Gamma \mathcal{F}(x)}$ for a.e. $x \in \mathbb{T}^{k}$. Hence, in this case $U(x)=T_{\Gamma \mathcal{F}(x)}\left(G^{1 / 2}(x)\right)^{\dagger}$ and therefore $U(\cdot): \mathbb{T}^{k} \rightarrow L\left(\mathbb{C}^{n}, \ell^{2}\left(\mathbb{Z}^{k}\right)\right)$ is a well defined measurable field of partial isometries. Then, $v_{j}: \mathbb{T}^{k} \rightarrow \ell^{2}\left(\mathbb{Z}^{k}\right)$ given by $v_{j}(x)=U(x) u_{j}(x) \in J_{\mathcal{W}}(x)$ for $j \in \mathbb{I}_{n}$ and $x \in \mathbb{T}^{k}$ are measurable vector fields such that $\left\{v_{j}(x)\right\}_{j \in \mathbb{I}_{r}}$ is an orthonormal system in $J_{\mathcal{W}}(x)$, for a.e. $x \in \mathbb{T}^{k}$; moreover, $\left[S_{E(\mathcal{F})}\right]_{x} v_{j}(x)=\lambda_{j}(x) v_{j}(x)$ for $j \in \mathbb{I}_{r}$ and a.e. $x \in \mathbb{T}^{k}$. Since $\operatorname{rk}\left[S_{E(\mathcal{F})}\right]_{x}=r$ for a.e. $x \in \mathbb{T}^{k}$, then we see that Eq. (18) holds in this case.

Finally, notice that if $E(\mathcal{F})$ is a frame for $\mathcal{W}$ then we should have that $r=\operatorname{rk}\left[S_{E(\mathcal{F})}\right]_{x}=\operatorname{dim} J_{\mathcal{W}}(x)$ for a.e. $x \in \mathbb{T}^{k}$ which shows the last part of the statement.

Remark 3.3. Let $\mathcal{F}=\left\{f_{i}\right\}_{i \in \mathbb{I}_{n}}$ be a finite sequence in $L^{2}\left(\mathbb{R}^{k}\right)$ such that $E(\mathcal{F})$ is a Bessel sequence. By Lemma 3.2 there exist measurable vectors fields $v_{j}: \mathbb{T}^{k} \rightarrow \ell^{2}\left(\mathbb{Z}^{k}\right)$ and measurable functions 
$\lambda_{j}: \mathbb{T}^{k} \rightarrow \mathbb{R}^{+}$such that they verify Eq. (18). In what follows we consider the fine spectral structure of $E(\mathcal{F})$ that is the weakly measurable function

$$
\mathbb{T}^{k} \ni x \mapsto\left(\lambda_{j}\left(\left[S_{E(\mathcal{F})}\right]_{x}\right)\right)_{j \in \mathbb{N}} \in\left(\ell_{+}^{1}\left(\mathbb{Z}^{k}\right)\right)^{\downarrow} \text { (non-increasing sequences) },
$$

where $\lambda_{j}\left(\left[S_{E(\mathcal{F})}\right]_{x}\right)=\lambda_{j}(x)$ for $j \in \mathbb{I}_{r(x)}$ and $\lambda_{j}\left(\left[S_{E(\mathcal{F})}\right]_{x}=0\right.$ for $j \geq r(x)+1$, for $x \in \mathbb{T}^{k}$. Hence, $\left(\lambda_{j}\left(\left[S_{E(\mathcal{F})}\right]_{x}\right)\right)_{j \in \mathbb{N}}$ coincides with the sequence of eigenvalues of the positive semidefinite finite rank operator $\left[S_{E(\mathcal{F})}\right]_{x}=S_{\Gamma \mathcal{F}(x)} \in L\left(\ell^{2}\left(\mathbb{Z}^{k}\right)\right)$, counting multiplicities and arranged in non-increasing order, for a.e. $x \in \mathbb{T}^{k}$.

Remark 3.4. Consider the notations from Definition 3.1. We now show that the right hand side in Eq. (17) is well defined. Indeed, by Lemma 3.2 we get a spectral representation of $\left[S_{E(\mathcal{F})}\right]_{(.)}$as in Eq. (18) in terms of the bounded and measurable functions $\lambda_{j}(\cdot): \mathbb{T}^{k} \rightarrow \mathbb{R}^{+}$, for $j \in \mathbb{I}_{n}$. If we consider the bounded and measurable function $d(x)=\operatorname{dim} J_{\mathcal{W}}(x) \geq r(x)$ for $x \in \mathbb{T}^{k}$ then, using Eq. (15) we see that

$$
P_{\varphi}^{J_{\mathcal{W}}(x)}(\Gamma \mathcal{F}(x))=\sum_{j \in \mathbb{I}_{r(x)}} \varphi\left(\lambda_{j}(x)\right)+(d(x)-r(x)) \varphi(0) \quad \text { for a.e. } x \in \mathbb{T}^{k} .
$$

Hence, the non-negative function

$$
\mathbb{T}^{k} \ni x \mapsto P_{\varphi}^{J_{\mathcal{W}}(x)}(\Gamma \mathcal{F}(x))
$$

is bounded and measurable and therefore integrable on $\mathbb{T}^{k}$. This shows that the convex potential $P_{\varphi}^{\mathcal{W}}(E(\mathcal{F}))$ is a well defined non-negative real number.

Incidentally, Remark 3.4 above shows that if $\varphi(0)=0$ then the convex potential $P_{\varphi}^{\mathcal{W}}=P_{\varphi}$ does not depend on the FSI subspace $\mathcal{W}$.

Example 3.5. Let $\mathcal{W}$ be a FSI subspace of $L^{2}\left(\mathbb{R}^{k}\right)$ and let $\mathcal{F}=\left\{f_{i}\right\}_{i \in \mathbb{I}_{n}} \in \mathcal{W}^{n}$. If we set $\varphi(x)=x^{2}$ for $x \in \mathbb{R}^{+}$then, the corresponding potential on $E(\mathcal{F})$, that we shall denote $\operatorname{FP}(E(\mathcal{F})$ ), is given by

$$
\operatorname{FP}(E(\mathcal{F}))=\int_{\mathbb{T}^{k}} \operatorname{tr}\left(S_{\Gamma \mathcal{F}(x)}^{2}\right) d x=\int_{\mathbb{T}^{k}} \sum_{i, j \in \mathbb{I}_{n}}\left|\left\langle\Gamma f_{i}(x), \Gamma f_{j}(x)\right\rangle\right|^{2} d x
$$

Hence, $\mathrm{FP}(E(\mathcal{F}))$ is a natural extension of the Benedetto-Fickus frame potential of Eq. (16).

Remark 3.6. Let $\mathcal{W}$ be a SI subspace of $L^{2}\left(\mathbb{R}^{k}\right)$ and let $A \in L\left(\ell^{2}\left(\mathbb{Z}^{k}\right)\right)^{+}$be a positive operator: in [21], E. Dutkay introduces the local trace function of $A$ relative to $\mathcal{W}$, denoted $\tau_{\mathcal{W}, A}: \mathbb{T}^{k} \rightarrow[0, \infty]$ as follows: for $x \in \mathbb{T}^{k}$,

$$
\tau_{\mathcal{W}, A}(x)=\operatorname{tr}\left(A\left[P_{\mathcal{W}}\right]_{x}\right),
$$

where $\operatorname{tr}(\cdot)$ denotes the usual (semi-finite) trace in $L\left(\ell^{2}\left(\mathbb{Z}^{k}\right)\right)$. We can extend the notion of local trace function as described above to the following setting: given $T \in L\left(L^{2}\left(\mathbb{R}^{k}\right)\right)^{+}$a positive and $\mathrm{SP}$ operator, we let the local trace function of $T$ with respect to the SI subspace $\mathcal{W}$ be given by

$$
\tau_{\mathcal{W}, T}(x)=\operatorname{tr}\left([T]_{x}\left[P_{\mathcal{W}}\right]_{x}\right), \quad x \in \mathbb{T}^{k}
$$

Notice that if $A \in L\left(\ell^{2}\left(\mathbb{Z}^{k}\right)\right)^{+}$and $T \in L\left(L^{2}\left(\mathbb{R}^{k}\right)\right)^{+}$is the unique positive and SP operator such that $[T]_{x}=A$ for $x \in \mathbb{T}^{k}$ then

$$
\tau_{\mathcal{W}, A}(x)=\tau_{\mathcal{W}, T}(x), \quad x \in \mathbb{T}^{k}
$$

If we assume further that $\mathcal{W}$ is a FSI subspace, we consider $\varphi \in \operatorname{Conv}\left(\mathbb{R}^{+}\right)$and take $\mathcal{F}=\left\{f_{i}\right\}_{i \in \mathbb{I}_{n}} \in$ $\mathcal{W}^{n}$ then

$$
P_{\varphi}^{\mathcal{W}}(E(\mathcal{F}))=\int_{\mathbb{T}^{k}} \tau_{\mathcal{W}, \varphi\left(S_{E(\mathcal{F})}\right)}(x) d x
$$


where $\varphi\left(S_{E(\mathcal{F})}\right) \in L\left(L^{2}\left(\mathbb{R}^{k}\right)\right)^{+}$is obtained by the functional calculus. Indeed, notice that in this case $\varphi\left(S_{E(\mathcal{F})}\right)$ is a SP operator such that

$$
\left[\varphi\left(S_{E(\mathcal{F})}\right)\right]_{x}=\varphi\left(\left[S_{E(\mathcal{F})}\right]_{x}\right)=\varphi\left(S_{\Gamma \mathcal{F}(x)}\right), \quad \text { for a.e. } x \in \mathbb{T}^{k} .
$$

Let $\mathcal{W}$ be a FSI subspace. In what follows we show that, under some natural restrictions, the convex potentials $P_{\varphi}^{\mathcal{W}}(E(\mathcal{F}))$ for finite sequences $\mathcal{F} \in \mathcal{W}^{n}$ detect tight frames for $\mathcal{W}$ as their minimizers (see Theorem 3.9 below). In turn, this last fact motivates the study of the structure of minimizers of convex potentials for finitely generated sequences in $L^{2}\left(\mathbb{R}^{k}\right)$ (under some restrictions) since these minimizers can be considered as natural substitutes of tight frames. In order to state the results on this matter, we introduce the following notions and notations.

Remark 3.7. Let $\left(X, \mathcal{X}, \mu_{X}\right),\left(Y, \mathcal{Y}, \mu_{Y}\right)$ be two measure spaces; we consider their direct sum, denoted $X \oplus Y$, given by the three-tuple $\left(X \oplus Y, \mathcal{X} \oplus \mathcal{Y}, \mu_{X} \oplus \mu_{Y}\right)$, where

1. $X \oplus Y=X \stackrel{d}{\cup} Y$ (the disjoint union of the sets); we further consider the canonical inclusions $\eta_{X}: X \rightarrow X \oplus Y$ and $\eta_{Y}: Y \rightarrow X \oplus Y$ of $X$ and $Y$ into their disjoint union; hence $\eta_{X}$ and $\eta_{Y}$ are injective functions such that $\eta_{X}(X) \cap \eta_{Y}(Y)=\emptyset$ and $\eta_{X}(X) \cup \eta_{Y}(Y)=X \oplus Y$.

2. $\mathcal{X} \oplus \mathcal{Y}=\left\{A \oplus B=\eta_{X}(A) \cup \eta_{Y}(B): A \in \mathcal{X}, B \in \mathcal{Y}\right\}$;

3. $\mu_{X} \oplus \mu_{Y}$ is the measure given by $\mu_{X} \oplus \mu_{Y}(A \oplus B)=\mu_{X}(A)+\mu_{Y}(B)$;

Notice that using the maps $\eta_{X}$ and $\eta_{Y}$ we can consider (as we sometimes do) $X, Y \subset X \oplus Y$. $\triangle$

Notations 3.8. In what follows we consider:

1. A FSI subspace of $L^{2}\left(\mathbb{R}^{k}\right)$ of length $\ell$, denoted $\mathcal{W}$;

2. $\mathcal{F}=\left\{f_{i}\right\}_{i \in \mathbb{I}_{n}} \in \mathcal{W}^{n}$ such that $E(\mathcal{F})$ is a Bessel sequence;

3. $d(x)=\operatorname{dim} J_{\mathcal{W}}(x) \leq \ell$, for $x \in \mathbb{T}^{k}$;

4. The Lebesgue measure on $\mathbb{R}^{k}$; denoted $|\cdot| ; X_{i}=d^{-1}(i) \subseteq \mathbb{T}^{k}$ and $p_{i}=\left|X_{i}\right|, i \in \mathbb{I}_{\ell}$.

5. We denote by $C_{\mathcal{W}}=\sum_{i \in \mathbb{I}_{\ell}} i \cdot p_{i}$.

6. The spectrum of $\mathcal{W}$ is the measurable $\operatorname{set} \operatorname{Spec}(\mathcal{W})=\bigcup_{i \in \mathbb{I}_{\ell}} X_{i}=\left\{x \in \mathbb{T}^{k}: d(x) \neq 0\right\}$.

Theorem 3.9 (Structure of $P_{\varphi}^{\mathcal{W}}$ minimizers with norm restrictions). Consider the Notations 3.8 and assume that $\sum_{i \in \mathbb{I}_{n}}\left\|f_{i}\right\|^{2}=1$. If $\varphi \in \operatorname{Conv}\left(\mathbb{R}^{+}\right)$, then

$$
P_{\varphi}^{\mathcal{W}}(E(\mathcal{F})) \geq C_{\mathcal{W}} \varphi\left(C_{\mathcal{W}}^{-1}\right)
$$

Moreover, if $\varphi \in \operatorname{Conv}_{\mathrm{s}}\left(\mathbb{R}^{+}\right)$then equality holds in (21) iff $E(\mathcal{F})$ is a tight frame for $\mathcal{W}$ i.e.

$$
S_{E(\mathcal{F})}=C_{\mathcal{W}}^{-1} P_{\mathcal{W}}
$$

Proof. Let $\left(X_{i j}, \mathcal{X}_{i j},|\cdot|_{i j}\right)$ where $X_{i j}=X_{i}, \mathcal{X}_{i j}=\mathcal{X}_{i}$ the $\sigma$-algebra of Lebesgue measurable sets in $X_{i}$ and $|\cdot|_{i j}=|\cdot|_{i}$ the Lebesgue measure in $X_{i}$, for $j \in \mathbb{I}_{i}$ and $i \in \mathbb{I}_{\ell}$. With the notations in Remark 3.7, we consider the measure space

$$
(X, \mathcal{X}, \mu)=\bigoplus_{i \in \mathbb{I}_{\ell}} \bigoplus_{j \in \mathbb{I}_{i}}\left(X_{i j}, \mathcal{X}_{i j},|\cdot|_{i j}\right)
$$


For $i \in \mathbb{I}_{\ell}$ and $j \in \mathbb{I}_{i}$ we further consider the canonical inclusions $\eta_{i, j}: X_{i, j} \rightarrow X$. Hence, for every $x \in X$ there exists unique $i \in \mathbb{I}_{\ell}, j \in \mathbb{I}_{i}$ and $\tilde{x} \in X_{i, j}=X_{i}$ such that $\eta_{i, j}(\tilde{x})=x$. Notice that by construction, $\mu(X)=\sum_{i \in \mathbb{I}_{\ell}} i \cdot p_{i}=C_{\mathcal{W}}$.

Let $\lambda_{E(\mathcal{F})}: X \rightarrow \mathbb{R}^{+}$be the measurable function of eigenvalues of $E(\mathcal{F})$ defined as follows: for $x \in X$, let $(i, j) \in \mathbb{I}_{\ell} \times \mathbb{I}_{i}$ and $\tilde{x} \in X_{i, j}=X_{i}$ be (uniquely determined) such that $\eta_{i, j}(\tilde{x})=x$; in this case we set

$$
\lambda_{E(\mathcal{F})}(x)=\lambda_{j}\left(\left[S_{E(\mathcal{F})}\right] \tilde{x}\right)=\lambda_{j}\left(S_{\Gamma \mathcal{F}(\tilde{x})}\right),
$$

where $\mathbb{T}^{k} \ni x \mapsto\left(\lambda_{j}\left(\left[S_{E(\mathcal{F})}\right]_{x}\right)\right)_{j \in \mathbb{N}} \in\left(\ell_{+}^{1}\left(\mathbb{Z}^{k}\right)\right)^{\downarrow}$ is the fine spectral structure of $E(\mathcal{F})$ defined in Remark 3.3. We claim that if $\varphi \in \operatorname{Conv}\left(\mathbb{R}^{+}\right)$, then

$$
P_{\varphi}^{\mathcal{W}}(E(\mathcal{F}))=\int_{X} \varphi\left(\lambda_{E(\mathcal{F})}(x)\right) d \mu(x) .
$$

Indeed, for Eq. (17)

$$
P_{\varphi}^{\mathcal{W}}(E(\mathcal{F}))=\int_{\mathbb{T}^{k}} P_{\varphi}^{J_{\mathcal{W}}(x)}(\Gamma \mathcal{F}(x)) d x=\int_{\operatorname{Spec}(\mathcal{W})} P_{\varphi}^{J_{\mathcal{W}}(x)}(\Gamma \mathcal{F}(x)) d x
$$

where $P_{\varphi}^{J_{\mathcal{W}}(x)}(\Gamma \mathcal{F}(x))$ is the convex potential associated with $\left(\varphi, J_{\mathcal{W}}(x)\right)$ of the finite sequence $\Gamma \mathcal{F}(x)=\left\{\Gamma f_{i}(x)\right\}_{i \in \mathbb{I}_{n}}$ in $\ell^{2}\left(\mathbb{Z}^{k}\right)$ as defined in Eq. (15) (notice that $P_{\varphi}^{J_{\mathcal{W}}(x)}(\Gamma \mathcal{F}(x))=0$ for $x \in$ $\left.\mathbb{T}^{k} \backslash \operatorname{Spec}(\mathcal{W})\right)$. Therefore, if $x \in X_{i}$ for some $i \in \mathbb{I}_{\ell}$ then

$$
P_{\varphi}^{J_{\mathcal{W}}(x)}(\Gamma \mathcal{F}(x))=\sum_{j=1}^{i} \varphi\left(\lambda_{j}\left(S_{\Gamma \mathcal{F}(x)}\right)\right)
$$

For $i \in \mathbb{I}_{\ell}$ we have that

$$
\int_{X_{i}} P_{\varphi}^{J_{\mathcal{W}}(x)}(\Gamma \mathcal{F}(x)) d x=\int_{X_{i}} \sum_{j=1}^{i} \varphi\left(\lambda_{j}\left(S_{\Gamma \mathcal{F}(x)}\right)\right) d x=\int_{\oplus_{j=1}^{i} X_{i j}} \varphi\left(\lambda_{E(\mathcal{F})}(x)\right) d \mu(x) .
$$

Therefore, since $\operatorname{Spec}(\mathcal{W})=\bigcup_{i \in \mathbb{I}_{\ell}} X_{i}$ and $X=\oplus_{i \in \mathbb{I}_{\ell}} \oplus_{j \in \mathbb{I}_{i}} X_{i, j}$,

$P_{\varphi}^{\mathcal{W}}(E(\mathcal{F}))=\sum_{i \in \mathbb{I}_{\ell}} \int_{X_{i}} P_{\varphi}^{J_{\mathcal{W}}(x)}(\Gamma \mathcal{F}(x)) d x=\sum_{i \in \mathbb{I}_{\ell}} \int_{\oplus_{j=1}^{i} X_{i j}} \varphi\left(\lambda_{E(\mathcal{F})}(x)\right) d \mu(x)=\int_{X} \varphi\left(\lambda_{E(\mathcal{F})}(x)\right) d \mu(x)$

which proves Eq. (23). In particular, if we take $\varphi(x)=x$ in Eq. (23) we get that

$$
\int_{X} \lambda_{E(\mathcal{F})}(x) d \mu(x)=\int_{\mathbb{T}^{k}} \operatorname{tr}\left(S_{\Gamma \mathcal{F}(x)}\right) d x=\int_{\mathbb{T}^{k}} \sum_{i \in \mathbb{I}_{n}}\left\|\Gamma f_{i}(x)\right\|^{2} d x=\sum_{i \in \mathbb{I}_{n}}\left\|f_{i}\right\|^{2}=1 .
$$

Consider the probability measure $\tilde{\mu}=C_{\mathcal{W}}^{-1} \mu$. Then, as in Example 2.5, we have that

$$
\int_{X} \lambda_{E(\mathcal{F})}(x) d \tilde{\mu}(x)=C_{\mathcal{W}}^{-1} \Longrightarrow C_{\mathcal{W}}^{-1} \cdot 1_{X} \prec \lambda_{E(\mathcal{F})} \quad(\text { in }(X, \mathcal{X}, \tilde{\mu}))
$$

If we let $\varphi \in \operatorname{Conv}\left(\mathbb{R}^{+}\right)$then, using the previous facts and Theorem 2.4, we get that

$$
\begin{aligned}
\varphi\left(C_{\mathcal{W}}^{-1}\right)=\int_{X} \varphi\left(C_{\mathcal{W}}^{-1} \cdot 1_{X}\right) d \tilde{\mu} & \stackrel{2.4}{\leq} \int_{X} \varphi\left(\lambda_{E(\mathcal{F})}(x)\right) d \tilde{\mu}(x) \\
& =C_{\mathcal{W}}^{-1} \int_{X} \varphi\left(\lambda_{E(\mathcal{F})}(x)\right) d \mu(x) \stackrel{(23)}{=} C_{\mathcal{W}}^{-1} P_{\varphi}^{\mathcal{W}}(E(\mathcal{F}))
\end{aligned}
$$


which proves Eq. (21). If $\varphi \in \operatorname{Conv}_{\mathrm{s}}\left(\mathbb{R}^{+}\right)$and also $P_{\varphi}^{\mathcal{W}}(E(\mathcal{F}))=\varphi\left(C_{\mathcal{W}}^{-1}\right) C_{\mathcal{W}}$, using Eq. (23), we get that

$$
\int_{X} \varphi\left(\lambda_{E(\mathcal{F})}(x)\right) d \tilde{\mu}(x)=\int_{X} \varphi\left(C_{\mathcal{W}}^{-1}\right) d \tilde{\mu} .
$$

Hence, by Proposition 2.6 and the majorization relation in Eq. (24),

$$
\left(\lambda_{E(\mathcal{F})}\right)^{*}=C_{\mathcal{W}}^{-1} 1_{[0,1]} \Longrightarrow \lambda_{i}\left(\left[S_{E(\mathcal{F})}\right]_{x}\right)=C_{\mathcal{W}}^{-1} \text { for } i \in \mathbb{I}_{d(x)} \text { and a.e. } x \in \mathbb{T}^{k} .
$$

Therefore, $S_{E(\mathcal{F})}=C_{\mathcal{W}}^{-1} P_{\mathcal{W}}$ i.e. $E(\mathcal{F})$ is a tight frame for $\mathcal{W}$. Conversely, notice that if $S_{E(\mathcal{F})}=$ $C_{\mathcal{W}}^{-1} P_{\mathcal{W}}$ then lower bound in Eq. (21) is attained.

\section{Fine spectral structure of shift generated oblique duals in FSI subspaces}

Throughout this section $\mathcal{V}, \mathcal{W} \subseteq L^{2}\left(\mathbb{R}^{k}\right)$ denote FSI subspaces such that $\mathcal{V} \oplus \mathcal{W}^{\perp}=L^{2}\left(\mathbb{R}^{k}\right)$ and $\mathcal{F}=\left\{f_{i}\right\}_{i \in \mathbb{I}_{n}} \in \mathcal{W}^{n}$ denotes a finite sequence such that $E(\mathcal{F})$ is a frame for $\mathcal{W}$.

Next we recall some characterizations of the condition $\mathcal{S} \oplus \mathcal{T}^{\perp}=L^{2}\left(\mathbb{R}^{k}\right)$ for SI subspaces and a characterization of shift generated $(\mathrm{SG})$ oblique duals of $E(\mathcal{F})$; these results together with [5] allow us to obtain the exact value of the aliasing norm corresponding to the consistent sampling induced by the FSI subspaces $\mathcal{V}$ and $\mathcal{W}$. In Section 4.2 we obtain a detailed description of the fine spectral structure (i.e. eigenvalues) of the frame operators of SG oblique $\mathcal{V}$-duals of the (fixed) frame $E(\mathcal{F})$ for $\mathcal{W}$. We will apply these results in Section 5, where we compute SG oblique dual frames with norm restrictions that simultaneously minimize the convex potentials $P_{\varphi}^{\mathcal{V}}$ for all $\varphi \in \operatorname{Conv}\left(\mathbb{R}^{+}\right)$.

\subsection{SG oblique duals and aliasing in FSI subspaces}

Following [28] (see also [17, 26, 29]) we consider the set of SG $\mathcal{V}$-duals of $E(\mathcal{F})$ :

$$
\mathcal{D}_{\mathcal{V}}^{S G}(\mathcal{F})=\mathcal{D}_{\mathcal{V}}^{S G}(E(\mathcal{F}))=\left\{E(\mathcal{G}) \in \mathcal{D}_{\mathcal{V}}(E(\mathcal{F})): \mathcal{G}=\left\{g_{i}\right\}_{i \in \mathbb{I}_{n}} \in \mathcal{V}^{n}\right\}
$$

In case $\mathcal{V}=\mathcal{W}$ then we write $\mathcal{D}^{S G}(\mathcal{F})=\mathcal{D}_{\mathcal{W}}^{S G}(E(\mathcal{F})$ ) (which is the class of SG duals of type I, in the terminology of [28]). Given $E(\mathcal{G}) \in \mathcal{D}_{\mathcal{V}}^{S G}(\mathcal{F})$ we obtain the following (structured) reconstruction formulas: for every $f \in \mathcal{W}$ and $g \in \mathcal{V}$,

$$
f=\sum_{(\ell, i) \in \mathbb{Z}^{k} \times \mathbb{I}_{n}}\left\langle f, T_{\ell} g_{i}\right\rangle T_{\ell} f_{i} \quad \text { and } \quad g=\sum_{(\ell, i) \in \mathbb{Z}^{k} \times \mathbb{I}_{n}}\left\langle g, T_{\ell} f_{i}\right\rangle T_{\ell} g_{i} .
$$

Next we describe some results related with the general assumption for studying oblique duality, namely $\mathcal{V} \oplus \mathcal{W}^{\perp}=L^{2}\left(\mathbb{R}^{k}\right)$, for the FSI subspaces $\mathcal{V}$ and $\mathcal{W}$, as well as SG oblique duality. The next two results can be derived using combinations of results and techniques in [2, 32, 33].

Lemma 4.1. With the previous notations and assumptions, let $J_{\mathcal{V}}$ and $J_{\mathcal{W}}$ denote the range functions of the $S I$ subspaces $\mathcal{V}$ and $\mathcal{W}$, respectively. Then,

1. $\mathcal{W}^{\perp}$ is a SI subspace with range function $J_{\mathcal{W}^{\perp}}(x)=\left[J_{\mathcal{W}}(x)\right]^{\perp}$ for a.e. $x \in \mathbb{T}^{k}$;

2. If $Q=P_{\mathcal{V} / / \mathcal{W}^{\perp}}$ then $Q$ is a shift preserving operator;

3. $J_{\mathcal{V}}(x) \oplus J_{\mathcal{W}}(x)^{\perp}=\ell^{2}\left(\mathbb{Z}^{k}\right)$ and $[Q]_{x}=P_{J_{\mathcal{V}}(x) / / J_{\mathcal{W}}(x)^{\perp}}$ for a.e. $x \in \mathbb{T}^{k}$.

4. $E(\mathcal{G}) \in \mathcal{D}_{\mathcal{V}}^{S G}(\mathcal{F}) \Longleftrightarrow \Gamma \mathcal{G}(x)$ is $J_{\mathcal{V}}(x)-$ dual of $\Gamma \mathcal{F}(x)$, for a.e $x \in \mathbb{T}^{k}$. 
Remark 4.2. Let $\mathcal{S}$ and $\mathcal{T}$ be closed subspaces of $L^{2}\left(\mathbb{R}^{k}\right)$. In order to characterize when the (algebraic) sum of these subspaces is a closed subspace we recall the Dixmier angle between $\mathcal{S}$ and $\mathcal{T}$, denoted by $[\mathcal{S}, \mathcal{T}]_{D} \in[0, \pi]$, given by

$$
\cos [\mathcal{S}, \mathcal{T}]_{D}=\sup \left\{|\langle v, w\rangle|, v \in \mathcal{S}_{1}, w \in \mathcal{T}_{1}\right\}
$$

where $\mathcal{S}_{1}=\{f \in \mathcal{S}:\|f\|=1\}$ (and similar for $\mathcal{T}_{1}$ ). It is well known (see [20]) that $[\mathcal{S}, \mathcal{T}]_{D}>0$ if and only if $\mathcal{S} \cap \mathcal{T}=\{0\}$ and $\mathcal{S}+\mathcal{T}$ is a closed subspace of $L^{2}\left(\mathbb{R}^{k}\right)$.

Assume further that $\mathcal{S} \oplus \mathcal{T}=L^{2}\left(\mathbb{R}^{k}\right)$ and let $Q=P_{\mathcal{S} / / \mathcal{T}}$ be the corresponding oblique projection. Then (see [20])

$$
\|Q\|=\frac{1}{\sin [\mathcal{S}, \mathcal{T}]_{D}} .
$$

Proposition 4.3. Let $\mathcal{S}, \mathcal{T} \subseteq L^{2}\left(\mathbb{R}^{k}\right)$ be SI subspaces of $L^{2}\left(\mathbb{R}^{k}\right)$. The following statements are equivalent:

1. $\mathcal{S} \oplus \mathcal{T}^{\perp}=L^{2}\left(\mathbb{R}^{k}\right)$;

2. $J_{\mathcal{S}}(x) \oplus J_{\mathcal{T}}(x)^{\perp}=\ell^{2}\left(\mathbb{Z}^{k}\right)$ for a.e. $x \in \mathbb{T}^{k}$ and $\operatorname{ess} \sup _{x \in \mathbb{T}^{k}}\left\|P_{J_{\mathcal{S}}(x) / / J_{\mathcal{T}}(x)^{\perp}}\right\|<\infty$;

3. $J_{\mathcal{S}}(x)^{\perp} \cap J_{\mathcal{T}}(x)=\{0\}$ and $\operatorname{essinf}_{x \in \mathbb{T}^{k}}\left[J_{\mathcal{S}}(x), J_{\mathcal{T}}(x)^{\perp}\right]_{D}>0$.

In this case we have that $\left[\mathcal{S}, \mathcal{T}^{\perp}\right]_{D}=\operatorname{ess}_{\inf }{ }_{x \in \mathbb{T}^{k}}\left[J_{\mathcal{S}}(x), J_{\mathcal{T}}(x)^{\perp}\right]_{D}$.

As an application of the previous results we compute the exact value of the aliasing norm (see $[22,30])$ in terms of the relative geometry of the FSI subspaces $\mathcal{V}$ and $\mathcal{W}$. Indeed, recall that the aliasing norm corresponding to the consistent sampling

$$
f \mapsto \tilde{f}=P_{\mathcal{W} / / \mathcal{V}^{\perp}} f, \quad \text { for } f \in L^{2}\left(\mathbb{R}^{k}\right)
$$

denoted $A(\mathcal{V}, \mathcal{W})$, is given by

$$
A(\mathcal{V}, \mathcal{W})=\sup _{e \in \mathcal{W}^{\perp}} \frac{\left\|P_{\mathcal{W} / / \mathcal{V}^{\perp}} e\right\|}{\|e\|}=\left\|P_{\mathcal{W} / / \mathcal{V}^{\perp}} P_{\mathcal{W}^{\perp}}\right\|
$$

The aliasing norm is a measure of the incidence of $\mathcal{W}^{\perp}$ in the consistent sampling induced by $P_{\mathcal{W} / \mathcal{V}^{\perp}}$ and it plays a role in applications of oblique duality.

Definition 4.4. Let $\mathcal{S}, \mathcal{T} \subset L^{2}\left(\mathbb{R}^{k}\right)$ be closed subspaces. We define the aperture between $\mathcal{S}$ and $\mathcal{T}$, denoted $[\mathcal{S}, \mathcal{T}]^{a} \in[0, \pi / 2]$, as the angle given by

$$
\cos \left([\mathcal{S}, \mathcal{T}]^{a}\right)=\inf _{f \in \mathcal{T},\|f\|=1}\left\|P_{\mathcal{S}} f\right\|
$$

Remark 4.5. With the notations of Definition 4.4, we point out that the aperture $[\mathcal{S}, \mathcal{T}]^{a}$ coincides with the notion of angle between the subspaces $\mathcal{S}$ and $\mathcal{T}$ as defined in [48. (and $\cos \left([\mathcal{S}, \mathcal{T}]^{a}\right.$ ) is also known as the infimum cosine angle from [32]). It is known that the following relation holds (see $[32,33]):$

$$
\cos \left([\mathcal{S}, \mathcal{T}]_{D}\right)^{2}=1-\cos \left([\mathcal{S}, \mathcal{T}]^{a}\right)^{2} \Longrightarrow[\mathcal{S}, \mathcal{T}]^{a}=\pi / 2-\left[\mathcal{S}, \mathcal{T}^{\perp}\right]_{D}
$$

Hence, using the relations above and Proposition 4.3 (see also [32]) we get that if $\mathcal{S}, \mathcal{T} \subset L^{2}\left(\mathbb{R}^{k}\right)$ are SI subspaces such that $\mathcal{S} \oplus \mathcal{T}^{\perp}=L^{2}\left(\mathbb{R}^{k}\right)$ then

$$
[\mathcal{S}, \mathcal{T}]^{a}=\operatorname{ess}_{\sup _{x \in \mathbb{T}^{k}}}\left[J_{\mathcal{S}}(x), J_{\mathcal{T}}(x)\right]^{a}<\pi / 2 .
$$


Consider again the notations of Definition 4.4 and assume further that $L^{2}\left(\mathbb{R}^{k}\right)=\mathcal{S} \oplus \mathcal{T}^{\perp}$. Then, using Remarks 4.2 and 4.5 we see that

$$
\left\|P_{\mathcal{S} / / \mathcal{T}^{\perp}}\right\|=\frac{1}{\sin \left[\mathcal{S}, \mathcal{T}^{\perp}\right]_{D}}=\frac{1}{\cos [\mathcal{S}, \mathcal{T}]^{a}} .
$$

From this we obtain the following upper bound for the aliasing norm (see [49])

$$
A(\mathcal{S}, \mathcal{T}) \leq\left\|P_{\mathcal{S} / / \mathcal{T}^{\perp}}\right\|=\frac{1}{\cos [\mathcal{S}, \mathcal{T}]^{a}}
$$

Notice that this known bound is not sharp; indeed, if we take $\mathcal{S}=\mathcal{T}$ then $A(\mathcal{S}, \mathcal{T})=0$ but $\cos [\mathcal{S}, \mathcal{T}]^{a}=1$.

Next we compute the exact value of the aliasing norm.

Proposition 4.6. With the previous notations and assumptions, the aliasing norm $A(\mathcal{V}, \mathcal{W})$ corresponding to the FSI oblique pair $(\mathcal{V}, \mathcal{W})$ is given by

$$
A(\mathcal{V}, \mathcal{W})=\tan \left([\mathcal{V}, \mathcal{W}]^{a}\right)
$$

Proof. Notice that by assumption $J_{\mathcal{V}}(x)$ and $J_{\mathcal{W}}(x)$ are finite dimensional subspaces of $\ell^{2}\left(\mathbb{Z}^{k}\right)$ and, by Proposition 4.3, we see that $J_{\mathcal{V}}(x)^{\perp} \oplus J_{\mathcal{W}}(x)=\ell^{2}\left(\mathbb{Z}^{k}\right)$, for a.e. $x \in \mathbb{T}^{k}$. Hence, we can apply the results from [5], and conclude that

$$
A\left(J_{\mathcal{V}}(x), J_{\mathcal{W}}(x)\right)=\left\|P_{J_{\mathcal{W}}(x) / / J_{\mathcal{V}}(x)^{\perp}} P_{J_{\mathcal{W}}(x)^{\perp}}\right\|=\tan \left(\left[J_{\mathcal{V}}(x), J_{\mathcal{W}}(x)\right]^{a}\right), \quad \text { for a.e. } x \in \mathbb{T}^{k} .
$$

Therefore, using Remark 4.5, we get that

$$
A(\mathcal{V}, \mathcal{W})=\left\|P_{\mathcal{W} / \mathcal{V}^{\perp}} P_{\mathcal{W} \perp}\right\|=\operatorname{ess}_{\sup _{x \in \mathbb{T}^{k}}} \tan \left(\left[J_{\mathcal{V}}(x), J_{\mathcal{W}}(x)\right]^{a}\right)=\tan \left([\mathcal{V}, \mathcal{W}]^{a}\right)
$$

Conjecture 4.7. We conjecture that Proposition 4.6 holds for the consistent sampling corresponding to an oblique decomposition $\mathcal{S} \oplus \mathcal{T}^{\perp}=\mathcal{H}$ in an arbitrary Hilbert space $\mathcal{H}$. By the results from [5] the conjecture holds for finite dimensional $\mathcal{S}$ and $\mathcal{T}$. By Proposition 4.6] this conjecture holds for some infinite dimensional subspaces $\mathcal{S}$ and $\mathcal{T}$ as well.

\subsection{Fine spectral structure of SG oblique duals}

Let $E(\mathcal{G}) \in \mathcal{D}_{\mathcal{V}}^{S G}(\mathcal{F})$ and let $S_{E(\mathcal{G})}$ denote the frame operator of $E(\mathcal{G})$. Recall that in this case $S_{E(\mathcal{G})}$ is a shift preserving (SP) operator such that $\left[S_{E(\mathcal{G})}\right]_{x}=S_{\Gamma \mathcal{G}(x)}$ for a.e. $x \in \mathbb{T}^{k}$ and the fine spectral structure of $E(\mathcal{G})$ is the function $\mathbb{T}^{k} \ni x \mapsto\left(\lambda_{j}\left(\left[S_{E(\mathcal{G})}\right]_{x}\right)\right)_{j \in \mathbb{N}} \in\left(\ell_{+}^{1}\left(\mathbb{Z}^{k}\right)\right)^{\downarrow}$, that consists of the sequence of eigenvalues of the positive finite rank operator $\left[S_{E(\mathcal{G})}\right]_{x}=S_{\Gamma \mathcal{G}(x)}$, counting multiplicities and arranged in non-increasing order for a.e. $x \in \mathbb{T}^{k}$ (see Remark 3.3).

In the next result we consider the measurable function $d: \mathbb{T}^{k} \rightarrow\{0, \ldots, n\}$ given by $d(x)=$ $\operatorname{dim} J_{\mathcal{W}}(x)=\operatorname{dim} J_{\mathcal{V}}(x)$ for a.e. $x \in \mathbb{T}^{k}$.

Lemma 4.8. Let $\mathcal{G}=\left\{g_{i}\right\}_{i \in \mathbb{I}_{n}} \in \mathcal{V}^{n}$ be such that $E(\mathcal{G})$ is a frame for $\mathcal{V}$. Let $B \in L\left(L^{2}\left(\mathbb{R}^{k}\right)\right)^{+}$be a shift preserving operator such that $R(B) \subseteq \mathcal{V}$. Then, there exists $\mathcal{Z}=\left\{z_{i}\right\}_{i \in \mathbb{I}_{n}} \in \mathcal{V}^{n}$ such that $B=S_{E(\mathcal{Z})}$ and $T_{E(\mathcal{G})} T_{E(\mathcal{Z})}^{*}=0$ if and only if $\operatorname{rk}\left([B]_{x}\right) \leq n-d(x)$ for a.e. $x \in \mathbb{T}^{k}$.

Proof. First notice that by considering a convenient finite partition of $\mathbb{T}^{k}$ into measurable sets we can assume, without loss of generality, that $d(x)=d \in \mathbb{N}$ for a.e. $x \in \mathbb{T}^{k}$. Notice that in this case 
$n \geq d$. By Lemma 3.2 there exist measurable vector fields $v_{j}: \mathbb{T}^{k} \rightarrow \ell^{2}\left(\mathbb{Z}^{k}\right)$ for $j \in \mathbb{I}_{n}$ such that, if $\mathbb{T}^{k} \ni x \mapsto\left(\lambda_{j}(x)\right)_{j \in \mathbb{N}} \in\left(\ell_{+}^{1}\left(\mathbb{Z}^{k}\right)\right)^{\downarrow}$ denotes the fine spectral structure of $E(\mathcal{G})$, then

$$
\left[S_{E(\mathcal{G})}\right]_{x}=\sum_{j \in \mathbb{I}_{d}} \lambda_{j}(x) v_{j}(x) \otimes v_{j}(x), \quad \text { for a.e. } x \in \mathbb{T}^{k} .
$$

Moreover, in this case $\left\{v_{j}(x)\right\}_{j \in \mathbb{I}_{d}}$ is an ONB of $J_{\mathcal{V}}(x)$ for a.e. $x \in \mathbb{T}^{k}$. Assume that $B \in L\left(L^{2}\left(\mathbb{R}^{k}\right)\right)^{+}$ is a shift preserving operator such that $R(B) \subseteq \mathcal{V}$ and such that $\operatorname{rk}\left([B]_{x}\right) \leq n-d$ for a.e. $x \in \mathbb{T}^{k}$. Since $[B]_{x} \in L\left(\ell^{2}\left(\mathbb{Z}^{k}\right)\right)^{+}$is such that $R\left([B]_{x}\right) \subseteq J_{\mathcal{V}}(x)$ for a.e. $x \in \mathbb{T}^{k}$ then, using the measurable vector fields $\left\{v_{j}\right\}_{j \in \mathbb{I}_{d}}$ as above (indeed, the measurable field of matrix representations of $[B]_{x}$ with respect to $\left\{v_{j}(x)\right\}_{j \in \mathbb{I}_{d}}$ and the results from [43]) we get measurable fields $w_{j}: \mathbb{T}^{k} \rightarrow \ell^{2}\left(\mathbb{Z}^{k}\right)$ for $j \in \mathbb{I}_{d}$, such that $\left\{w_{j}(x)\right\}_{j \in \mathbb{I}_{d}}$ is an ONB of $J_{\mathcal{V}}(x)$ and $[B]_{x} w_{j}(x)=\lambda_{j}\left([B]_{x}\right) w_{j}(x)$ for $j \in \mathbb{I}_{d}$ and a.e. $x \in \mathbb{T}^{k}$. In particular, we see that

$$
[B]_{x}^{1 / 2}=\sum_{j=1}^{\min \{d, n-d\}} \lambda_{j}\left([B]_{x}\right)^{1 / 2} w_{j}(x) \otimes w_{j}(x), \quad \text { for a.e. } x \in \mathbb{T}^{k} .
$$

Consider the measurable field of positive semidefinite matrices $G_{\mathcal{G}}: \mathbb{T}^{k} \rightarrow \mathcal{M}_{n}(\mathbb{C})$ given by $G_{\mathcal{G}}(x)=$ $\left(\left\langle\Gamma g_{i}(x), \Gamma g_{j}(x)\right\rangle\right)_{i, j \in \mathbb{I}_{n}}, x \in \mathbb{T}^{k}$. Again by [43], there exist measurable field of vectors $u_{j}: \mathbb{T}^{k} \rightarrow \mathbb{C}^{n}$ for $j \in \mathbb{I}_{n}$ such that for a.e. $x \in \mathbb{T}^{k}$ we have that $\left\{u_{j}(x)\right\}_{j \in \mathbb{I}_{n}}$ is an ONB of $\mathbb{C}^{n}, G_{\mathcal{G}}(x) u_{j}(x)=$ $\lambda_{j}(x) u_{j}(x)$ for $j \in \mathbb{I}_{d}$ and $G_{\mathcal{G}}(x) u_{j}(x)=0$ for $d+1 \leq j \leq n$ (since $G_{\mathcal{G}}(x)$ and $\left[S_{E(\mathcal{G})}\right]_{x}$ have the same strictly positive eigenvalues).

Let $V: \mathbb{T}^{k} \rightarrow L\left(\mathbb{C}^{n}, \ell^{2}\left(\mathbb{Z}^{k}\right)\right)$ be the measurable field of partial isometries given by

$$
V(x) u_{j}(x)=\left\{\begin{array}{ccc}
w_{j-d} & \text { if } & d+1 \leq j \leq d+\min \{d, n-d\} \\
0 & \text { otherwise }
\end{array}\right.
$$

Hence $V(x) V^{*}(x)$ is the orthogonal projection onto $\operatorname{span}\left\{w_{j}(x): j \in \mathbb{I}_{\min \{d, n-d\}}\right\}$ and thus $[B]_{x} V(x) V^{*}(x)=[B]_{x}$ for a.e. $x \in \mathbb{T}^{k}$; on the other hand, $R\left(V^{*}(x)\right)=\operatorname{ker} V(x)^{\perp} \subseteq \operatorname{span}\left\{u_{j}(x)\right.$ : $d+1 \leq j \leq n\} \Longrightarrow T_{\Gamma \mathcal{G}(x)} V^{*}(x)=0$ for a.e. $x \in \mathbb{T}^{k}$.

For $i \in \mathbb{I}_{n}$ we set $z_{i} \in \mathcal{V}$ determined uniquely by $\Gamma z_{i}(x)=[B]_{x}^{1 / 2} V(x) e_{i}$ for a.e. $x \in \mathbb{T}^{k}$, where $\left\{e_{i}\right\}_{i \in \mathbb{I}_{n}}$ denotes the canonical ONB of $\mathbb{C}^{n}$. If we set $\mathcal{Z}=\left\{z_{i}\right\}_{i \in \mathbb{I}_{n}}$ then $T_{\Gamma \mathcal{Z}(x)}=\left[B^{1 / 2}\right]_{x} V(x)$ for a.e. $x \in \mathbb{T}^{k}$; hence, using Eq. (10), we see that

$$
\left[T_{E(\mathcal{G})} T_{E(\mathcal{Z})}^{*}\right]_{x}=T_{\Gamma \mathcal{G}(x)} T_{\Gamma \mathcal{Z}(x)}^{*}=T_{\Gamma \mathcal{G}(x)} V^{*}(x)\left[B^{1 / 2}\right]_{x}=0, \quad \text { for a.e. } x \in \mathbb{T}^{k} .
$$

On the other hand, notice that

$$
\left[S_{E(\mathcal{Z})}\right]_{x}=S_{\Gamma \mathcal{Z}(x)}=[B]_{x}^{1 / 2} V(x) V^{*}(x)[B]_{x}^{1 / 2}=[B]_{x}, \quad \text { for a.e. } x \in \mathbb{T}^{k} .
$$

Conversely, assume that there exists $\mathcal{Z}=\left\{z_{i}\right\}_{i \in \mathbb{I}_{n}} \in \mathcal{V}^{n}$ such that $B=S_{E(\mathcal{Z})}$ and $T_{E(\mathcal{G})} T_{E(\mathcal{Z})}^{*}=0$. Then, by Eq. (10), we get that $0=T_{\Gamma \mathcal{G}(x)} T_{\Gamma \mathcal{Z}(x)}^{*}$ and hence $\operatorname{rk}\left(T_{\Gamma \mathcal{Z}(x)}^{*}\right) \leq n-\operatorname{rk}\left(T_{\Gamma \mathcal{G}(x)}\right)=n-d(x)$ for a.e. $x \in \mathbb{T}^{k}$. Therefore,

$$
\operatorname{rk}\left[S_{E(\mathcal{Z})}\right]_{x}=\operatorname{rk}\left(S_{\Gamma \mathcal{Z}(x)}\right)=\operatorname{rk}\left(T_{\Gamma \mathcal{Z}(x)}\right)=\operatorname{rk}\left(T_{\Gamma \mathcal{Z}(x)}^{*}\right) \leq n-d(x) \quad \text { for a.e. } x \in \mathbb{T}^{k} .
$$

Definition 4.9. Let $\mathcal{G}=\left\{g_{i}\right\}_{i \in \mathbb{I}_{n}}$ be such that $E(\mathcal{G})$ is a frame for $\mathcal{V}$ with frame operator $A=S_{E(\mathcal{G})}$. Recall that $d(x)=\operatorname{dim} J_{\mathcal{V}}(x)$ for $x \in \mathbb{T}^{k}$. Then, we consider

$$
U_{\mathcal{V}}(E(\mathcal{G}))=\left\{A+B: B \in L\left(L^{2}\left(\mathbb{R}^{k}\right)\right)^{+} \text {is } \mathrm{SP}, R(B) \subset \mathcal{V}, \operatorname{rk}\left([B]_{x}\right) \leq n-d(x) \text {, for a.e. } x \in \mathbb{T}^{k}\right\} .
$$


Proposition 4.10. Let $E(\mathcal{F})_{\mathcal{V}}^{\#}=\left\{T_{\ell} f_{\mathcal{V}, i}^{\#}\right\}_{(\ell, i) \in \mathbb{Z}^{k} \times \mathbb{I}_{n}}$ denote the canonical $\mathcal{V}$-dual of $\mathcal{F}$. Then,

$$
\left\{S_{E(\mathcal{G})}: E(\mathcal{G}) \in \mathcal{D}_{\mathcal{V}}^{S G}(\mathcal{F})\right\}=U_{\mathcal{V}}\left(E(\mathcal{F})_{\mathcal{V}}^{\#}\right)
$$

Proof. Let $\mathcal{G}=\left\{g_{i}\right\}_{i \in \mathbb{I}_{n}} \in \mathcal{V}^{n}$ be such that $E(\mathcal{G}) \in \mathcal{D}_{\mathcal{V}}^{S G}(\mathcal{F})$. Let $\mathcal{Z}=\left\{z_{i}\right\}_{i \in \mathbb{I}_{n}} \in \mathcal{V}^{n}$ be given by $z_{i}=g_{i}-f_{\mathcal{V}, i}^{\#}$ for $i \in \mathbb{I}_{n}$. Then $E(\mathcal{Z})=\left\{T_{\ell} z_{i}\right\}_{(\ell, i) \in \mathbb{Z}^{k} \times \mathbb{I}_{n}}$ is a Bessel sequence in $\mathcal{V}$ such that $T_{E(\mathcal{G})}=T_{E(\mathcal{F})_{\mathcal{V}}^{\#}}+T_{E(\mathcal{Z})}$. In this case we have that $T_{E(\mathcal{Z})} T_{E(\mathcal{F})}^{*}=0$ and therefore $T_{E(\mathcal{Z})} T_{E(\mathcal{F})_{\mathcal{V}}^{*}}^{*}=0$, since $R\left(T_{E(\mathcal{F})}^{*}\right)=R\left(T_{E(\mathcal{F})_{\mathcal{V}}^{*}}^{*}\right.$. Thus,

$$
S_{E(\mathcal{G})}=\left(T_{E(\mathcal{F})_{\mathcal{V}}^{\#}}+T_{E(\mathcal{Z})}\right)\left(T_{E(\mathcal{F})_{\mathcal{V}}^{\#}}+T_{E(\mathcal{Z})}\right)^{*}=S_{E(\mathcal{F})_{\mathcal{V}}^{\#}}+S_{E(\mathcal{Z})}
$$

We conclude that $B=S_{E(\mathcal{Z})} \in L\left(L^{2}\left(\mathbb{R}^{k}\right)\right)^{+}$is SP, $R\left(S_{E(\mathcal{Z})}\right) \subset \mathcal{V}$ and, by Lemma 4.8, that $\operatorname{rk}\left[S_{E(\mathcal{Z})}\right]_{x} \leq n-d(x)$ for a.e. $x \in \mathbb{T}^{k}$.

Conversely, if $S \in U_{\mathcal{V}}\left(E(\mathcal{F})_{\mathcal{V}}^{\#}\right)$ then $S=S_{E(\mathcal{F})_{\mathcal{V}}^{\#}}+B$, where $B \in L\left(L^{2}\left(\mathbb{R}^{k}\right)\right)^{+}$is $\mathrm{SP}, R(B) \subset \mathcal{V}$ and $\operatorname{rk}\left([B]_{x}\right) \leq n-d(x)$ for a.e. $x \in X$. By Lemma 4.8 we see that there exists $\mathcal{Z}=\left\{z_{i}\right\}_{i \in \mathbb{I}_{n}}$ such that $T_{E(\mathcal{Z})} T_{E(\mathcal{F})}^{*}=0$ and $B=S_{E(\mathcal{Z})}$. If we let $\mathcal{G}=\left\{g_{i}\right\}_{i \in \mathbb{I}_{n}}$ be given by $g_{i}=f_{\mathcal{V}, i}^{\#}+z_{i}$ for $i \in \mathbb{I}_{n}$, then $E(\mathcal{G})$ is a Bessel sequence in $\mathcal{V}$ such that $T_{E(\mathcal{G})}=T_{E(\mathcal{F})_{\mathcal{V}}^{\#}}+T_{E(\mathcal{Z})}$. Using that $T_{E(\mathcal{Z})} T_{E(\mathcal{F})_{\mathcal{V}}^{*}}^{*}=0$ we conclude, as before, that

$$
S_{E(\mathcal{G})}=S_{E(\mathcal{F})_{\mathcal{V}}^{\#}}+S_{E(\mathcal{Z})}=S_{E(\mathcal{F})_{\mathcal{V}}^{\#}}+B=S
$$

Proposition 4.10 shows that the set of frame operators of SG $\mathcal{V}$-duals of a fixed frame $\mathcal{F}$ can be described in terms of the additive model $U_{\mathcal{V}}\left(E(\mathcal{F})_{\mathcal{V}}^{\#}\right)$ introduced in Definition 4.9, It turns out that the fine spectral structure of the elements of $U_{\mathcal{V}}\left(E(\mathcal{F})_{\mathcal{V}}^{\#}\right)$ can be described using a natural extension of the Fan-Pall interlacing theorem for measurable fields of positive matrices. We develop both results in the Appendix section (see Theorems 6.3 and 6.4). As a consequence we obtain the following

Theorem 4.11 (Fine spectral structure of $\mathcal{V}$-duals). Let $E(\mathcal{F})_{\mathcal{V}}^{\#}$ be the canonical $\mathcal{V}$-dual frame of $E(\mathcal{F})$. Denote the fine spectral structure of $E(\mathcal{F})_{\mathcal{V}}^{\#}$ by $\mathbb{T}^{k} \ni x \mapsto\left(\lambda_{\mathcal{V}, i}^{\#}(x)\right)_{i \in \mathbb{N}}, x \in \mathbb{T}^{k}$. Let $m$ be the measurable function given by $m(x)=2 d(x)-n$, for $x \in \mathbb{T}^{k}$. Given a measurable function $\mu: \mathbb{T}^{k} \rightarrow\left(\ell^{1}(\mathbb{N})^{+}\right)^{\downarrow}$ (decreasing sequences) described as $\mu=\left(\mu_{i}\right)_{i \in \mathbb{N}}$, the following are equivalent:

1. There exists $E(\mathcal{G}) \in \mathcal{D}_{\mathcal{V}}^{S G}(\mathcal{F})$ such that $\mu(x)=\lambda\left(\left[S_{E(\mathcal{G})}\right]_{x}\right)=\lambda\left(S_{\Gamma \mathcal{G}(x)}\right)$ for every $x \in \mathbb{T}^{k}$.

2. For a.e. $x \notin \operatorname{Spec}(\mathcal{V}), \mu(x)=0$. For a.e. $x \in \operatorname{Spec}(\mathcal{V}), \mu_{i}(x)=0$ for $i \geq d(x)+1$ and

(a) in case that $m(x) \leq 0$, then $\mu_{i}(x) \geqslant \lambda_{\mathcal{V}, i}^{\#}(x)$ for $i \in \mathbb{I}_{d(x)}$;

(b) in case that $m(x) \geq 1$, then $\mu_{i}(x) \geqslant \lambda_{\mathcal{V}, i}^{\#}(x)$ for $i \in \mathbb{I}_{d(x)}$ and

$$
\mu_{n-d(x)+i}(x)=\mu_{d(x)-m(x)+i}(x) \leq \lambda_{\mathcal{V}, i}^{\#}(x) \quad \text { for } \quad i \in \mathbb{I}_{m(x)} .
$$

Proof. It follows from Proposition 4.10 and Theorem 6.4.

As a consequence of the description of the fine spectral structure of $\mathcal{V}$-duals of $E(\mathcal{F})$ we characterize the existence of tight $\mathcal{V}$-duals of $E(\mathcal{F})$ that are shift generated (compare with [25]).

Corollary 4.12. With the notations of Theorem 4.11 then there exists a c-tight $\mathcal{V}$-dual $E(\mathcal{G}) \in$ $\mathcal{D}_{\mathcal{V}}^{S G}(\mathcal{F})$ if and only if

$$
\text { 1. } S_{E(\mathcal{F})_{\mathcal{V}}^{\#}} \leq c \cdot P_{\mathcal{V}}
$$


2. $\operatorname{rk}\left(\left[c \cdot P_{\mathcal{V}}-S_{E(\mathcal{F})_{\mathcal{V}}^{\#}}\right]_{x}\right) \leq \min \{d(x), n-d(x)\}$ for a.e. $x \in \operatorname{Spec}(\mathcal{V})$.

Proof. Theorem 4.11 imply that there exists a $\mathcal{V}$-dual $E(\mathcal{G}) \in \mathcal{D}_{\mathcal{V}}^{S G}(\mathcal{F})$ such that $S_{E(\mathcal{G})}=c \cdot P_{\mathcal{V}}$ if and only if $c \geq \lambda_{\mathcal{V}, i}^{\#}(x)$ for $i \in \mathbb{I}_{d(x)}$ and $\lambda_{\mathcal{V}, i}^{\#}(x)=c$ for $i \in \mathbb{I}_{m(x)}$ whenever $m(x)=2 d(x)-n \geq 1$, for a.e. $x \in \operatorname{Spec}(\mathcal{V})$. These last two conditions are equivalent to the fact that $c \cdot P_{\mathcal{V}} \geq S_{E(\mathcal{F})_{\mathcal{V}}^{\#}}$ and

$$
\operatorname{rk}\left(\left[c \cdot P_{\mathcal{V}}-S_{E(\mathcal{F})_{\mathcal{V}}^{\#}}\right]_{x}\right) \leq d(x)-m(x)=n-d(x) \quad \text { whenever } \quad m(x) \geq 1 .
$$

Also notice that in case $m(x) \leq 0$ then $n-d(x) \geq d(x)=\operatorname{dim} J_{\mathcal{V}}(x)$. The proof follows from these remarks.

Remark 4.13. Consider the notations of Theorem 4.11. As a consequence of Corollary 4.12, we get the following dichotomy related with the existence of tight oblique $\mathcal{V}$-duals of $E(\mathcal{F})$ :

1. If $n \geq 2 d(x)$ for a.e. $x \in \mathbb{T}^{k}$ then for every $c \geq\left\|S_{E(\mathcal{F})_{\mathcal{V}}^{\#}}\right\|$ there exists $E(\mathcal{G}) \in \mathcal{D}_{\mathcal{V}}^{S G}(\mathcal{F})$ that is a $c$-tight frame for $\mathcal{V}$.

2. If there exists $N \subseteq \mathbb{T}^{k}$ with positive Lebesgue measure such that $n<2 d(x)$ for a.e. $x \in N$ and there exists a $c$-tight frame $E(\mathcal{G}) \in \mathcal{D}_{\mathcal{V}}^{S G}(\mathcal{F})$ then $c=\left\|S_{E(\mathcal{F})_{\mathcal{V}}^{\#}}\right\|$.

\section{Applications: optimal oblique SG-duals with norm restrictions}

As before, we consider two FSI subspaces $\mathcal{V}$ and $\mathcal{W}$ such that $\mathcal{W}^{\perp} \oplus \mathcal{V}=L^{2}\left(\mathbb{R}^{k}\right)$ and $\mathcal{F}=\left\{f_{i}\right\}_{i \in \mathbb{I}_{n}} \in$ $\mathcal{W}^{n}$ such that $E(\mathcal{F})$ is a frame for $\mathcal{W}$.

As a consequence of the description of the fine spectral structure of elements in $\mathcal{D}_{\mathcal{V}}^{S G}(\mathcal{F})$, we see that the canonical $\mathcal{V}$-dual is optimal with respect to several criteria. Nevertheless, in applied situations, the canonical dual might not be the best choice: for example, we can be interested in duals of $E(\mathcal{F})$ such that the spectrum of their frame operators are as concentrated as possible. Ideally, we would search for tight dual frames for $E(\mathcal{F})$, although Corollary 4.12 shows that there are restrictions for the existence of such duals.

In order to search for alternate $\mathcal{V}$-duals that are spectrally more stable, we proceed as follows: for $w \geq \sum_{i \in \mathbb{I}_{n}}\left\|f_{\mathcal{V}, i}^{\#}\right\|^{2}$, where $E(\mathcal{F})_{\mathcal{V}}^{\#}=\left\{T_{\ell} f_{\mathcal{V}, i}^{\#}\right\}_{(\ell, i) \in \mathbb{Z}^{k} \times \mathbb{I}_{n}}$, we consider

$$
\mathcal{D}_{\mathcal{V}, w}^{S G}(\mathcal{F})=\mathcal{D}_{\mathcal{V}, w}^{S G}(E(\mathcal{F})) \stackrel{\text { def }}{=}\left\{E(\mathcal{G}) \in \mathcal{D}_{\mathcal{V}}^{S G}(\mathcal{F}): \mathcal{G}=\left\{g_{i}\right\}_{i \in \mathbb{I}_{n}} \text { and } \sum_{i \in \mathbb{I}_{n}}\left\|g_{i}\right\|^{2} \geq w\right\}
$$

Notice that if $w>\sum_{i \in \mathbb{I}_{n}}\left\|f_{\mathcal{V}, i}^{\#}\right\|^{2}$ then $E(\mathcal{F})_{\mathcal{V}}^{\#} \notin \mathcal{D}_{\mathcal{V}, w}^{S G}(\mathcal{F})$ and therefore, it is natural to ask whether there is an optimal dual fulfilling the previous requirements. Using the identity

$$
\sum_{i \in \mathbb{I}_{n}}\left\|g_{i}\right\|^{2}=\int_{\mathbb{T}^{k}} \sum_{i \in \mathbb{I}_{n}}\left\|\Gamma g_{i}(x)\right\|^{2} d x=\int_{\mathbb{T}^{k}} \operatorname{tr}\left(\left[S_{E(\mathcal{G})}\right]_{x}\right) d x=\int_{\mathbb{T}^{k}} \sum_{i \in \mathbb{N}} \mu_{i}(x) d x
$$

where $\lambda\left(\left[S_{E(\mathcal{G})}\right]_{x}\right)=\left(\mu_{i}(x)\right)_{i \in \mathbb{N}}$ for a.e. $x \in \mathbb{T}^{k}$, we see that Theorem 4.11 gives a complete solution to a frame design problem in the sense that it allows to get a complete description of the eigenvalue lists of the frame operators of elements in $\mathcal{D}_{\mathcal{V}, w}^{S G}(\mathcal{F})$. It is then natural to seek for those oblique SG-duals $E(\mathcal{G}) \in \mathcal{D}_{\mathcal{V}, w}^{S G}(\mathcal{F})$ that minimize the convex potentials $P_{\varphi}^{\mathcal{V}}$, for $\varphi \in \operatorname{Conv}\left(\mathbb{R}^{+}\right)$; in order to deal with this problem we first examine a construction known as water-filling in terms of submajorization, in the general context of measure spaces (see Theorem 5.5). We then apply these results together with the properties of submajorization and results from matrix analysis to conclude that there are structural optimal duals with norm restrictions. These optimal solutions are obtained in terms of a non-commutative water-filling construction. 


\subsection{Water-filling in measure spaces}

The water-filling construction goes back to the work of Shanon [46], as the solution of an optimal spectral allocation problem (see [19]). The water-filling strategy has also been the main tool in the design of channels with optimal capacity (see 47] and the more recent work on iterative water-filling techniques [41, 45]).

As a first step towards an extension of this construction, we examine its scalar counter-part in the general context of measure spaces. In the next section we show that the water-filling technique produces optimal solutions in the general (non-commutative) context of measurable fields of positive semidefinite matrix valued functions.

Throughout this section the triple $(X, \mathcal{X}, \mu)$ denotes a probability space. Recall that we denote by $L^{\infty}(X, \mu)^{+}=\left\{f \in L^{\infty}(X, \mu): f \geq 0\right\}$.

Definition 5.1 (Water-filling at level $c$ ). Let $f \in L^{\infty}(X, \mu)^{+}$. Given $c \geq \operatorname{essinf} f \geq 0$ we consider $f_{c} \in L^{\infty}(X, \mu)^{+}$given by $f_{c}=\max \{f, c\}=f+(c-f)^{+}$, where $g^{+}$denotes the positive part of a real function $g$.

In order to study the submajorization properties of the function $f_{c}$ obtained by the water-filling construction as above, we consider the following result in which we obtain a simple relation between the decreasing rearrangements of $f$ and $f_{c}$.

Lemma 5.2. Let $f \in L^{\infty}(X, \mu)^{+}$and let $c \geq \operatorname{ess} \inf f \geq 0$. Consider the number

$$
s_{0}=\mu\{x \in X: f(x)>c\} . \quad \text { Then } \quad f_{c}^{*}(s)=\left\{\begin{array}{cll}
f^{*}(s) & \text { if } & 0 \leq s<s_{0} \\
c & \text { if } & s_{0} \leq s \leq 1
\end{array}\right.
$$

Proof. Notice that by Eq. (11), for $0 \leq s<s_{0}$ we have that

$$
\begin{aligned}
f^{*}(s) & =\sup \left\{t \in \mathbb{R}^{+}: \mu\{x \in X: f(x)>t\}>s\right\} \\
& =\sup \left\{t \in \mathbb{R}^{+}: \mu\{x \in X: f(x)>t\}>s \text { and } t \geq c\right\} \\
& =\sup \left\{t \in \mathbb{R}^{+}: \mu\left\{x \in X: f_{c}(x)>t\right\}>s \text { and } t \geq c\right\} \\
& =\sup \left\{t \in \mathbb{R}^{+}: \mu\left\{x \in X: f_{c}(x)>t\right\}>s\right\}=f_{c}^{*}(s) .
\end{aligned}
$$

It is straightforward to see that if $s_{0} \leq s \leq 1$ then $f_{c}^{*}(s)=c$.

In order to prove Theorem 5.5 below, we shall need an explicit statement of some re-parametrized versions of the basics results of section 2.3 .

Lemma 5.3. Let $a, b \in \mathbb{R}$ be such that $a<b$ and let $k \in L^{\infty}([a, b], \nu)^{+}$be a non-increasing right continuous function, where $\nu=(b-a)^{-1} d t$ is the normalized Lebesgue measure on $[a, b]$. Then

1. The decreasing rearrangement $k^{*}(t)=k((b-a) t+a)$ for every $t \in[0,1)$.

2. Fix a constant $c \in \mathbb{R}$. Then

$$
(b-a) c \leq \int_{a}^{b} k(t) d t \Longrightarrow(s-a) c \leq \int_{a}^{s} k(t) d t \quad \text { for every } \quad s \in[a, b] .
$$

Proof. Straightforward.

With the notations of Lemma 5.3 above, notice that item 2. is a restatement (using the reparametrization from item 1) of the submajorization inequalities corresponding to $c \prec_{w} k$ in $([a, b], \nu)$ whenever $c \leq \int_{[a, b]} k d \nu$ (see Example 2.5). 
Remark 5.4. Let $f \in L^{\infty}(X, \mu)^{+}$and consider $\phi:[\operatorname{essinf} f, \infty) \rightarrow \mathbb{R}^{+}$given by

$$
\phi(c)=\int_{X} f_{c} d \mu=\int_{X} f(x)+(c-f(x))^{+} d \mu(x) .
$$

Then, it is easy to see that $\phi$ has the following properties:

1. $\phi(\operatorname{essinf} f)=\int_{X} f d \mu$ and $\lim _{c \rightarrow+\infty} \phi(c)=+\infty$;

2. $\phi$ is continuous and strictly increasing.

Hence, for every $w \geq \int_{X} f d \mu$ there exists a unique $c(w)=c \geq \operatorname{essinf} f \operatorname{such}$ that

$$
\phi(c(w))=w \quad \text { i.e. } \quad \int_{X} f_{c(w)} d \mu=w .
$$

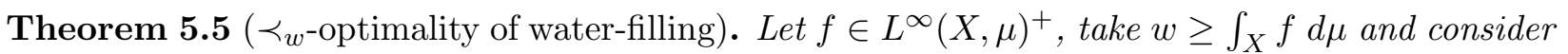
the constant $c(w)=c$ as in Remark 5.4. Then, for every $h \in L^{\infty}(X, \mu)^{+}$,

$$
f \leq h \quad \text { and } \quad \int_{X} h d \mu \geq w \Longrightarrow f_{c} \prec w h .
$$

Proof. Assume that $f \leq h$ and $\int_{X} h d \mu \geq w$. If we let $s_{0}=\mu\{x \in X: f(x)>c\}$ then, by Lemma [5.2, we have that Eq. (31) holds. Thus, using Remark 2.2, we see that if $0 \leq s<s_{0}$ then

$$
\int_{0}^{s} f_{c}^{*}(t) d t=\int_{0}^{s} f^{*}(t) d t \leq \int_{0}^{s} h^{*}(t) d t
$$

Fix now $s_{0} \leq s \leq 1$ and consider

$$
\alpha=\int_{0}^{s_{0}} h^{*}(t) d t-\int_{0}^{s_{0}} f_{c}^{*}(t) d t \geq 0 \quad \text { and } \quad k=h^{*}+\frac{1}{1-s_{0}} \alpha \in L^{\infty}([0,1], d t)^{+} .
$$

Notice that $k$ is a non-increasing right continuous map. In this case we get that

$$
\begin{aligned}
\int_{s_{0}}^{1} k(t) d t & =\int_{s_{0}}^{1} h^{*}(t) d t+\alpha \\
& =\int_{s_{0}}^{1} h^{*}(t) d t+\left(\int_{0}^{s_{0}} h^{*}(t) d t-\int_{0}^{s_{0}} f_{c}^{*}(t) d t\right) \\
& \geq w-\left(w-\left(1-s_{0}\right) c\right)=\left(1-s_{0}\right) c
\end{aligned}
$$

Then, Lemma 5.3 (applied to the map $\left.k\right|_{\left[s_{0}, 1\right]}$ ) implies that

$$
\left(s-s_{0}\right) c \leq \int_{s_{0}}^{s} k(t) d t=\int_{s_{0}}^{s} h^{*}(t) d t+\frac{s-s_{0}}{1-s_{0}} \alpha \quad \text { for every } \quad s \in\left[s_{0}, 1\right] .
$$

Hence, using the inequality above and Lemma 5.2, we conclude that for $s_{0} \leq s<1$

$$
\begin{aligned}
\int_{0}^{s} f_{c}^{*}(t) d t & =\int_{0}^{s_{0}} h^{*}(t) d t-\alpha+\left(s-s_{0}\right) c \\
& \leq \int_{0}^{s} h^{*}(t) d t+\left(\frac{s-s_{0}}{1-s_{0}}-1\right) \alpha \leq \int_{0}^{s} h^{*}(t) d t .
\end{aligned}
$$

This last fact together with Eq. (33) show that $f_{c} \prec{ }_{w} h$. 
Theorem 5.5 above implies a family of integral inequalities in terms of convex functions involving the water-filling of a function $f$ at level $c$. We will need these facts in order to show the optimality properties of the non-commutative version of waterfilling.

Corollary 5.6. With the notations of Theorem [5.5, if $\varphi \in \operatorname{Conv}\left(\mathbb{R}^{+}\right)$is non-decreasing then

$$
\int_{X} \varphi \circ h d \mu \geq \int_{X} \varphi \circ f_{c} d \mu \text {. }
$$

If there is a non-decreasing $\varphi \in \operatorname{Conv}_{\mathbf{s}}\left(\mathbb{R}^{+}\right)$such that equality holds in Eq. (34) then $h=f_{c}$.

Proof. The first claim is a consequence of the submajorization relation in Theorem 5.5 and Theorem 2.4. If we further assume that $\varphi \in \operatorname{Conv}_{\mathrm{s}}\left(\mathbb{R}^{+}\right)$is such that equality holds in Eq. (34) then, by Proposition 2.6, we see that $f_{c}^{*}=h^{*}$. Let $B=\{x \in X: f(x)>c\}$ so that $s_{0}=\mu(B)$. Then, it is straightforward to show that

$$
\left(f \cdot 1_{B}\right)^{*}(s)=\left\{\begin{array}{cll}
f^{*}(s) & \text { if } & s \in\left[0, s_{0}\right) \\
0 & \text { if } & s \in\left[s_{0}, 1\right)
\end{array}\right.
$$

Notice that, in particular, $\left(f \cdot 1_{B}\right)^{*}=1_{\left[0, s_{0}\right)} \cdot f_{c}^{*}$. On the other hand we have $\left(h \cdot 1_{B}\right)^{*}=1_{\left[0, s_{0}\right)} \cdot\left(h \cdot 1_{B}\right)^{*}$. Hence, since $h \geq h \cdot 1_{B} \geq f \cdot 1_{B}$, by Remark 2.2 we have that

$$
h^{*} \geq\left(h \cdot 1_{B}\right)^{*}=1_{\left[0, s_{0}\right)} \cdot\left(h \cdot 1_{B}\right)^{*} \geq\left(f \cdot 1_{B}\right)^{*}=1_{\left[0, s_{0}\right)} \cdot f_{c}^{*} \quad \Longrightarrow \quad\left(h \cdot 1_{B}\right)^{*}=\left(f \cdot 1_{B}\right)^{*} .
$$

Therefore, again by Remark 2.2, we get that $h \cdot 1_{B}=f \cdot 1_{B}=f_{c} \cdot 1_{B}>c \cdot 1_{B}$, where the last facts follow from Definition 5.1. Finally, notice that

$$
\mu\left(h^{-1}(\{c\})\right)=\left|\left(h^{*}\right)^{-1}(\{c\})\right|=\left|\left(f_{c}^{*}\right)^{-1}(\{c\})\right|=1-\mu(B),
$$

which shows that $h \cdot 1_{X \backslash B}=c \cdot 1_{X \backslash B}$ and hence $h=f_{c}$.

\subsection{Optimal SG-duals with norm restrictions: NC water-filling}

In what follows we show the existence of structural optimal SG oblique duals of a fixed frame $E(\mathcal{F})$ with norm restrictions, as described at the beginning of Section 5. That is, we explicitly construct a dual frame $E\left(\mathcal{G}^{\text {op }}\right) \in \mathcal{D}_{\mathcal{V}, w}^{S G}(\mathcal{F})$ such that for every $E(\mathcal{G}) \in \mathcal{D}_{\mathcal{V}, w}^{S G}(\mathcal{F})$ then

$$
P_{\varphi}^{\mathcal{V}}\left(E\left(\mathcal{G}^{\mathrm{op}}\right)\right) \leq P_{\varphi}^{\mathcal{V}}(E(\mathcal{G}))
$$

for every convex potential $P_{\varphi}^{\mathcal{V}}$ associated to a non-decreasing $\varphi \in \operatorname{Conv}\left(\mathbb{R}^{+}\right)$. Moreover, the arguments involved in this construction show that (structural) optimal SG oblique duals with norm restrictions share several spectral properties. We end the section with a non-commutative counterpart of the water-filling construction for functions, that allows to describe the spectral and geometrical structure of optimal $\mathrm{SG}$ oblique duals in $\mathcal{D}_{\mathcal{V}, w}^{S G}(\mathcal{F})$ in some detail.

Theorem 5.7 (Optimal duals in $\mathcal{D}_{\mathcal{V}, w}^{S G}(\mathcal{F})$ ). Let $\mathcal{V}$ and $\mathcal{W}$ be FSI subspaces of $L^{2}\left(\mathbb{R}^{k}\right)$ such that $\mathcal{W}^{\perp} \oplus \mathcal{V}=L^{2}\left(\mathbb{R}^{k}\right)$. Let $\mathcal{F}=\left\{f_{i}\right\}_{i \in \mathbb{I}_{n}}$ be such that $E(\mathcal{F})$ is a frame for $\mathcal{W}$ and $w>\sum_{i \in \mathbb{I}_{n}}\left\|f_{\mathcal{V}, i}^{\#}\right\|^{2}$, where $E(\mathcal{F})_{\mathcal{V}}^{\#}=\left\{T_{\ell} f_{\mathcal{V}, i}^{\#}\right\}_{(\ell, i) \in \mathbb{Z}^{k} \times \mathbb{I}_{n}}$. Then, there exists $\mathcal{G}^{\mathrm{op}}=\left\{g_{i}^{\mathrm{op}}\right\}_{i \in \mathbb{I}_{n}} \in \mathcal{V}^{n}$ such that:

1. $E\left(\mathcal{G}^{\mathrm{op}}\right) \in \mathcal{D}_{\mathcal{V}, w}^{S G}(\mathcal{F})$ and $\sum_{i \in \mathbb{I}_{n}}\left\|g_{i}^{\mathrm{op}}\right\|^{2}=w$.

2. For every $\mathcal{G}=\left\{g_{i}\right\}_{i \in \mathbb{I}_{n}}$ such that $E(\mathcal{G}) \in \mathcal{D}_{\mathcal{V}, w}^{S G}(\mathcal{F})$ and every non-decreasing $\varphi \in \operatorname{Conv}\left(\mathbb{R}^{+}\right)$ we have that

$$
P_{\varphi}^{\mathcal{V}}\left(E\left(\mathcal{G}^{\mathrm{op}}\right)\right) \leq P_{\varphi}^{\mathcal{V}}(E(\mathcal{G}))
$$


Proof. Let $d(x)=\operatorname{dim} J_{\mathcal{V}}(x)=\operatorname{dim} J_{\mathcal{W}}(x)$ for $x \in \mathbb{T}^{k}$. For each $i \in \mathbb{I}_{n}$, let $X_{i}=d^{-1}(i) \subseteq \mathbb{T}^{k}$, $p_{i}=\left|X_{i}\right|$ (the Lebesgue measure of $X_{i}$ ) and $r_{i}=\min \{n-i, i\}$. Since $E(\mathcal{F})$ is a frame for $\mathcal{W}$ then $\operatorname{Spec}(\mathcal{V})=\operatorname{Spec}(\mathcal{W})=\cup_{i \in \mathbb{I}_{n}} X_{i}$. Also, for $i \in \mathbb{I}_{n}$ and $j \in \mathbb{I}_{r_{i}}$ we consider the measure space $\left(X_{i j}, \mathcal{X}_{i j},|\cdot|_{i j}\right)$, where $X_{i j}=X_{i}, \mathcal{X}_{i j}=\mathcal{X}_{i}$ denotes the $\sigma$-algebra of Lebesgue measurable sets in $X_{i}$ and $|\cdot|_{i j}=|\cdot|_{i}$ denotes the Lebesgue measure in $X_{i}$. Then, using Remark 3.7, we construct the measure space

$$
(Y, \mathcal{Y}, \nu)=\bigoplus_{i \in \mathbb{I}_{n}} \bigoplus_{j \in \mathbb{I}_{r_{i}}}\left(X_{i j}, \mathcal{Y}_{i j},|\cdot|_{i j}\right)
$$

In particular, $\nu(Y)=\sum_{i \in \mathbb{I}_{n}} r_{i} \cdot p_{i}$. We further consider the canonical inclusion maps $\eta_{i, j}: X_{i, j} \rightarrow Y$ for $i \in \mathbb{I}_{n}$ and $j \in \mathbb{I}_{r_{i}}$.

Let $\mathcal{G}=\left\{g_{i}\right\}_{i \in \mathbb{I}_{n}}$ be such that $E(\mathcal{G}) \in \mathcal{D}_{\mathcal{V}}^{S G}(\mathcal{F})$. We shall denote by $A=S_{E(\mathcal{F})_{\mathcal{V}}^{\#}}$ and $S=S_{E(\mathcal{G})} \in$ $L\left(L^{2}\left(\mathbb{R}^{k}\right)\right)^{+}$. By Proposition 4.10, $S=S_{E(\mathcal{F})_{\nu}^{\#}}+B=A+B$, for some $B \in L\left(L^{2}\left(\mathbb{R}^{k}\right)\right)^{+}$which is $\mathrm{SP}, R(B) \subset \mathcal{V}$ and $\operatorname{rk}\left([B]_{x}\right) \leq n-d(x)$ for a.e. $x \in \mathbb{T}^{k}$. Let $i \in \mathbb{I}_{n}$; using Lidskii's additive inequality (see [4]) we get that for a.e. $x \in X_{i}$

$$
\left(\lambda_{i-j+1}\left([A]_{x}\right)+\lambda_{j}\left([B]_{x}\right)\right)_{j \in \mathbb{I}_{i}} \prec\left(\lambda_{j}\left([S]_{x}\right)\right)_{j \in \mathbb{I}_{i}},
$$

while $\lambda_{j}\left([S]_{x}\right)=0$ for $j \geq i+1$. Notice that $R\left([B]_{x}\right) \subset J_{\mathcal{V}}(x)$ and $\operatorname{rk}\left([B]_{x}\right) \leq n-i$. Therefore $\operatorname{rk}\left([B]_{x}\right) \leq \min \{n-i, i\}=r_{i}$. Then, for $x \in X_{i}$ we have that

$$
\lambda_{i-j+1}\left([A]_{x}\right)+\lambda_{j}\left([B]_{x}\right)=\left\{\begin{array}{ccc}
\lambda_{i-j+1}\left([A]_{x}\right)+\lambda_{j}\left([B]_{x}\right) & \text { if } & 1 \leq j \leq r_{i} \\
\lambda_{i-j+1}\left([A]_{x}\right) & \text { if } & r_{i}+1 \leq j \leq i
\end{array}\right.
$$

Now, Eq. (35) together with Eq. (36) imply that, for any $\varphi \in \operatorname{Conv}\left(\mathbb{R}^{+}\right)$: for a.e. $x \in X_{i}$ then

$$
\sum_{j=1}^{r_{i}} \varphi\left(\lambda_{i-j+1}\left([A]_{x}\right)+\lambda_{j}\left([B]_{x}\right)\right)+\sum_{j=r_{i}+1}^{i} \varphi\left(\lambda_{i-j+1}\left([A]_{x}\right)\right) \leq \sum_{j \in \mathbb{I}_{i}} \varphi\left(\lambda_{j}\left([S]_{x}\right)\right) .
$$

With the previous notations, we now consider the measurable function $h: Y \rightarrow \mathbb{R}^{+}$defined as follows: for $x \in Y$, let $(i, j) \in \mathbb{I}_{n} \times \mathbb{I}_{r_{i}}$ and $\tilde{x} \in X_{i, j}=X_{i}$ be (uniquely determined) such that $\eta_{i, j}(\tilde{x})=x$; in this case we set $h(x)=\lambda_{i-j+1}\left([A]_{\tilde{x}}\right)+\lambda_{j}\left([B]_{\tilde{x}}\right)$. If we let $w_{0}=\sum_{i \in \mathbb{I}_{n}}\left\|f_{\mathcal{V}, i}^{\#}\right\|^{2}$ and we assume that $E(\mathcal{G}) \in \mathcal{D}_{\mathcal{V}, w}^{S G}(\mathcal{F})$ then, using Eq. (30) we see that

$$
\int_{\mathbb{T}^{k}} \operatorname{tr}\left([B]_{x}\right) d x=\int_{\mathbb{T}^{k}} \operatorname{tr}\left([S]_{x}-[A]_{x}\right) d x \geq w-w_{0} \geq 0 .
$$

Consider now the measurable function $f: Y \rightarrow \mathbb{R}^{+}$given by $f(x)=\lambda_{i-j+1}\left([A]_{\tilde{x}}\right)$ for $\tilde{x} \in X_{i j}=X_{i}$, with $(i, j) \in \mathbb{I}_{n} \times \mathbb{I}_{r_{i}}$ such that $\eta_{i, j}(\tilde{x})=x$. Arguing as in the proof of Theorem 3.9 we get that

$$
\int_{Y} f d \nu=\sum_{i \in \mathbb{I}_{n}} \int_{X_{i}} \sum_{j \in \mathbb{I}_{r_{i}}} \lambda_{i-j+1}\left([A]_{x}\right) d x .
$$

Moreover, the previous facts show that if $E(\mathcal{G}) \in \mathcal{D}_{\mathcal{V}, w}^{S G}(\mathcal{F})$ we have that $h \geq f$ and

$$
\int_{Y} h(x) d \nu=\sum_{i \in \mathbb{I}_{n}} \sum_{j \in \mathbb{I}_{r_{i}}} \int_{X_{i}}\left(\lambda_{i-j+1}\left([A]_{x}\right)+\lambda_{j}\left([B]_{x}\right)\right) d x \geq\left(w-w_{0}\right)+\int_{Y} f(x) d \nu \stackrel{\text { def }}{=} w^{\prime} .
$$

Let $c=c\left(\frac{w^{\prime}}{\nu(Y)}\right)$ be as in Remark 5.4 and consider $f_{c}$ as in Definition [5.1, both with respect to the probability space $(Y, \mathcal{Y}, \tilde{\nu})$, where $\tilde{\nu}=\nu(Y)^{-1} \nu$. By Corollary 5.6 and the previous remarks we see that if $\varphi \in \operatorname{Conv}\left(\mathbb{R}^{+}\right)$is non-decreasing then

$$
\int_{Y} \varphi \circ f_{c} d \nu \leq \int_{Y} \varphi \circ h d \nu
$$


For $j \in \mathbb{I}_{n}$ we consider the measurable functions $\xi_{j}: \operatorname{Spec}(\mathcal{V}) \rightarrow \mathbb{R}^{+}$defined as follows: for $i \in \mathbb{I}_{n}$ and $x \in X_{i}$,

$$
\xi_{j}(x)=\left\{\begin{array}{ccc}
f_{c}\left(\eta_{i j}(x)\right)=\max \left\{c, \lambda_{i-j+1}\left([A]_{x}\right)\right\} & \text { if } & 1 \leq j \leq r_{i} \\
\lambda_{i-j+1}\left([A]_{x}\right) & \text { if } & r_{i}+1 \leq j \leq i \\
0 & \text { if } & i+1 \leq j \leq n
\end{array},\right.
$$

Notice that by construction, if $\varphi \in \operatorname{Conv}\left(\mathbb{R}^{+}\right)$then

$$
\int_{Y} \varphi \circ f_{c} d \nu=\sum_{i \in \mathbb{I}_{n}} \int_{X_{i}} \sum_{j \in \mathbb{I}_{r_{i}}} \varphi\left(\xi_{j}(x)\right) d x .
$$

Using the definition of $f$ and the properties of $f_{c}$ from Remark 5.4, we see that if $x \in X_{i}$, then there exist $\lambda_{1}^{\mathrm{op}}(x) \geq \ldots \geq \lambda_{r_{i}}^{\mathrm{op}}(x) \geq 0$ such that

$$
\xi_{j}(x)=\lambda_{i-j+1}\left([A]_{x}\right)+\lambda_{j}^{\mathrm{op}}(x) \quad \text { for every } \quad j \leq r_{i} .
$$

Let $\mu=\left(\mu_{j}\right)_{j \in \mathbb{N}}: \mathbb{T}^{k} \rightarrow \ell^{1}(\mathbb{N})^{+}$such that: $\mu_{j}(x)=0$ for $j \in \mathbb{N}$ whenever $x \in \mathbb{T}^{k} \backslash \operatorname{Spec}(\mathcal{V})$, while for $i \in \mathbb{I}_{n}$ and $x \in X_{i}$ then $\mu_{j}(x)=0$ for $j \geq i+1$ and

$$
\left(\mu_{j}(x)\right)_{j \in \mathbb{I}_{i}}=\left[\left(\xi_{j}(x)\right)_{j \in \mathbb{I}_{i}}\right]^{\downarrow} .
$$

Putting the previous remarks together we see that $\mu=\left(\mu_{j}\right)_{j \in \mathbb{N}}$ satisfies the conditions of item 2 in Theorem 4.11. Thus, there exists $\mathcal{G}^{\mathrm{op}}=\left\{g_{i}^{\mathrm{op}}\right\}_{i \in \mathbb{I}_{n}}$ such that $E\left(\mathcal{G}^{\mathrm{op}}\right) \in \mathcal{D}_{\mathcal{V}}^{S G}(\mathcal{F})$ and $\lambda\left(\left[S_{E\left(\mathcal{G}^{\mathrm{op}}\right)}\right]_{x}\right)=$ $\left(\mu_{j}(x)\right)_{j \in \mathbb{N}}$ for a.e. $x \in \mathbb{T}^{k}$. In this case, if we consider Eq. (30), use Eqs. (42), (43) and we take $\varphi(x)=x$ in Eq. (41) we have that

$$
\begin{aligned}
\sum_{i \in \mathbb{I}_{n}}\left\|g_{i}^{\mathrm{op}}\right\|^{2} & =\int_{\mathbb{T}^{k}} \sum_{j \in \mathbb{N}} \mu_{j}(x) d x=\sum_{i \in \mathbb{I}_{n}} \int_{X_{i}}\left(\sum_{j \in \mathbb{I}_{r_{i}}} \xi_{j}(x)+\sum_{j=r_{i}+1}^{i} \lambda_{i-j+1}\left([A]_{x}\right)\right) d x \\
& =\int_{Y} f_{c} d \nu+\sum_{i \in \mathbb{I}_{n}} \int_{X_{i}} \sum_{j=r_{i}+1}^{i} \lambda_{i-j+1}\left([A]_{x}\right) d x \\
& =\left(w-w_{0}\right)+\int_{\mathbb{T}^{k}} \operatorname{tr}\left([A]_{x}\right) d x=w
\end{aligned}
$$

where we have also used the relation in Eq. (38) above. In particular, $\mathcal{G}^{\text {op }}$ satisfies item 1 . in the statement. Now, if $E(\mathcal{G}) \in \mathcal{D}_{\mathcal{V}, w}^{S G}(\mathcal{F})$, using Eqs. (37), (39), (40) and (43) then,

$$
\begin{aligned}
P_{\varphi}^{\mathcal{V}}(E(\mathcal{G})) & \geq \int_{Y} \varphi \circ h d \nu+\sum_{i \in \mathbb{I}_{n}} \int_{X_{i}} \sum_{j=r_{i}+1}^{i} \varphi \circ \lambda_{i-j+1}\left([A]_{x}\right) d x \\
& \geq \int_{Y} \varphi \circ f_{c} d \nu+\sum_{i \in \mathbb{I}_{n}} \int_{X_{i}} \sum_{j=r_{i}+1}^{i} \varphi \circ \lambda_{i-j+1}\left([A]_{x}\right) d x=P_{\varphi}^{\mathcal{V}}\left(E\left(\mathcal{G}^{\mathrm{op}}\right)\right)
\end{aligned}
$$

where we have also used Eq. (41) and the fact that $\lambda\left(\left[\left(S_{E\left(\mathcal{G}^{\circ \mathrm{p}}\right)}\right]_{x}\right)=\mu(x)\right.$ for a.e. $x \in \mathbb{T}^{k}$.

Corollary 5.8 (Essential uniqueness of optimal $\mathcal{V}$-duals with norm restrictions). With the notations of Theorem 5.7 and its proof, assume that $\mathcal{G}=\left\{g_{i}\right\}_{i \in \mathbb{I}_{n}}$ is such that $E(\mathcal{G}) \in \mathcal{D}_{\mathcal{V}, w}^{S G}(\mathcal{F})$ and that there exists a non-decreasing $\varphi \in \operatorname{Conv}_{\mathrm{s}}\left(\mathbb{R}^{+}\right)$such that

$$
P_{\varphi}^{\mathcal{V}}\left(E\left(\mathcal{G}^{\mathrm{op}}\right)\right)=P_{\varphi}^{\mathcal{V}}(E(\mathcal{G})) .
$$

Let $B \in L\left(L^{2}\left(\mathbb{R}^{k}\right)\right)^{+}$be $S P$, with $R(B) \subset \mathcal{V}$ and such that $S_{E(\mathcal{G})}=S_{E(\mathcal{F})_{\mathcal{V}}^{\#}}+B=A+B$. Then, 
1. $\sum_{i \in \mathbb{I}_{n}}\left\|g_{i}\right\|^{2}=w$;

2. There exist $c>0$ and measurable vector fields $v_{i}: \mathbb{T}^{k} \rightarrow \ell^{2}\left(\mathbb{Z}^{k}\right)$ for $i \in \mathbb{I}_{n}$ such that $\left\{v_{i}(x)\right\}_{i \in \mathbb{I}_{d(x)}}$ is an $O N B$ of $J_{\mathcal{V}}(x)$ for a.e. $x \in \operatorname{Spec}(\mathcal{V})$,

$$
\left[S_{E(\mathcal{F})_{\mathcal{V}}^{\#}}\right]_{x}=[A]_{x}=\sum_{i \in \mathbb{I}_{d(x)}} \lambda_{i}\left([A]_{x}\right) v_{i}(x) \otimes v_{i}(x), \quad \text { for a.e. } x \in \operatorname{Spec}(\mathcal{V})
$$

and such that for a.e. $x \in \operatorname{Spec}(\mathcal{V})$ we have that

$$
[B]_{x}=\sum_{i=r(x)+1}^{d(x)}\left(c-\lambda_{i}\left([A]_{x}\right)\right)^{+} v_{i}(x) \otimes v_{i}(x),
$$

where $r(x)=\max \{2 d(x)-n, 0\}$, for $x \in \mathbb{T}^{k}$.

The constant $c\left(=c\left(\frac{w^{\prime}}{\nu(Y)}\right)\right)>0$ does not depend on $\mathcal{G}$. Moreover, in this case $P_{\psi}^{\mathcal{V}}(E(\mathcal{G}))=$ $P_{\psi}^{\mathcal{V}}\left(E\left(\mathcal{G}^{\mathrm{op}}\right)\right)$ for every non-decreasing $\psi \in \operatorname{Conv}\left(\mathbb{R}^{+}\right)$.

Proof. We use the notions and notations from the proof of Theorem [5.7. Arguing as in the last part of the proof of Theorem [5.7 we see that Eq. (44) implies that

$$
\int_{Y} \varphi \circ h d \tilde{\nu}=\int_{Y} \varphi \circ f_{c} d \tilde{\nu}
$$

where $\tilde{\nu}=\nu(Y)^{-1} \nu$ is the probability measure obtained by normalization of $\nu$. By Corollary 5.6 we get that $h=f_{c}$, where $c=c\left(\frac{w^{\prime}}{\nu(Y)}\right)$ is as in the proof of Theorem 5.7. Therefore, for $i \in \mathbb{I}_{n}$ and $x \in X_{i}$, then

$$
\lambda_{i-j+1}\left([A]_{x}\right)+\lambda_{j}\left([B]_{x}\right)=\left\{\begin{array}{ccc}
\max \left\{\lambda_{i-j+1}\left([A]_{x}\right), c\right\} & \text { if } & 1 \leq j \leq r_{i} \\
\lambda_{i-j+1}\left([A]_{x}\right) & \text { if } & r_{i}+1 \leq j \leq i
\end{array}\right.
$$

Moreover, by Eq. (37) and the properties of $h$ we have that

$$
\begin{aligned}
P_{\varphi}^{\mathcal{V}}(E(\mathcal{G})) & =\sum_{i \in \mathbb{I}_{n}} \int_{X_{i}} \sum_{j \in \mathbb{I}_{i}} \varphi\left(\lambda_{j}\left(\left[S_{E(\mathcal{G})}\right]_{x}\right)\right) d x \\
& \geq \int_{Y} \varphi \circ h d \nu+\sum_{i \in \mathbb{I}_{n}} \int_{X_{i}} \sum_{j=r_{i}+1}^{i} \varphi \circ \lambda_{i-j+1}\left([A]_{x}\right) d x=P_{\varphi}^{\mathcal{V}}(E(\mathcal{G})) .
\end{aligned}
$$

Therefore, we should have equality Eq. (37) for a.e. $x \in X_{i}$ and $i \in \mathbb{I}_{n}$. Since $\varphi$ is strictly convex, then the majorization relation in Eq. (35) together with the case of equality in Lidskii's inequality (see the Appendix section in [39]) imply that for $i \in \mathbb{I}_{n}$ and a.e. $x \in X_{i}$ there exists an ONB $\left\{z_{j}(x)\right\}_{j \in \mathbb{I}_{i}}$ of $J_{\mathcal{V}}(x)$ (but not necessarily of measurable vector fields as functions of $x$ ) such that

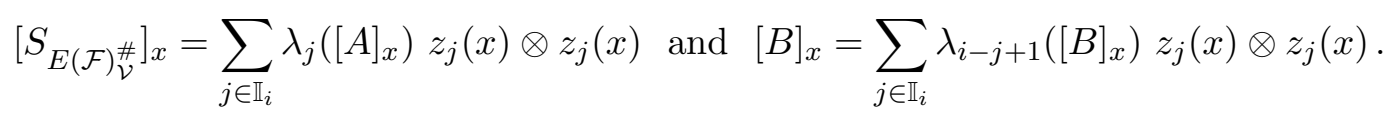

Let $P \in L\left(L^{2}\left(\mathbb{R}^{k}\right)\right)^{+}$denote the orthogonal projection onto $\mathcal{R}=\overline{R(B)}$, so that $P$ is SP and $[P]_{x}=$ $P_{R\left([B]_{x}\right)}$ for every $x \in \mathbb{T}^{k}$. Let $p: \mathbb{T}^{k} \rightarrow \mathbb{N}$ be the measurable function given by $p(x)=\operatorname{tr}\left([P]_{x}\right)$ for $x \in \mathbb{T}^{k}$. Then, by inspection of Eqs. (45) and (46) we see that $P S_{E(\mathcal{F})_{\mathcal{V}}^{\#}}=S_{E(\mathcal{F})_{\mathcal{V}}^{\#}} P$,

$$
[P]_{x}[A]_{x}[P]_{x}+[B]_{x}=c \cdot[P]_{x}, \quad \text { for a.e. } x \in \mathbb{T}^{k}
$$


and, for $i \in \mathbb{I}_{n}$ and $x \in X_{i}$,

$$
[I-P]_{x}[A]_{x}[I-P]_{x}=\sum_{j=1}^{i-p(x)} \lambda_{j}\left([A]_{x}\right) z_{j}(x) \otimes z_{j}(x)
$$

Since $a+(c-a)^{+}=\max \{a, c\}$ for $a, c \geq 0$, these last facts imply the existence of measurable vector fields $v_{i}: \mathbb{T}^{k} \rightarrow \ell^{2}\left(\mathbb{Z}^{k}\right)$ for $i \in \mathbb{I}_{n}$ with the desired properties; indeed, the previous identities show that we just have to consider measurable fields of eigenvectors of the operators $P S_{E(\mathcal{F})_{V}^{\#}} P$ and $(I-P) S_{E(\mathcal{F})_{\mathcal{V}}^{\#}}(I-P)$, whose existence follow from Lemma 3.2.

Finally, if $\mathcal{G}^{\text {op }}$ is as in Theorem [5.7, a careful inspection of the proof of that theorem shows that

$$
\lambda\left(\left[S_{E(\mathcal{G})}\right]_{x}\right)=\lambda\left(\left[S_{E\left(\mathcal{G}^{\mathrm{op}}\right)}\right]_{x}\right), \quad \text { for a.e. } x \in \operatorname{Spec}(\mathcal{V}),
$$

which implies the optimality properties of $E(\mathcal{G})$ for a non-decreasing $\psi \in \operatorname{Conv}\left(\mathbb{R}^{+}\right)$.

Notice that with the notations of Corollary 5.8 above, we see that for a.e. $x \in \mathbb{T}^{k}$ then

$$
\left[S_{E\left(\mathcal{G}^{\mathrm{op}}\right)}\right]_{x}=\sum_{i=1}^{r(x)} \lambda_{i}\left([A]_{x}\right) v_{i}(x) \otimes v_{i}(x)+\sum_{i=r(x)+1}^{d(x)} \max \left\{\lambda_{i}\left([A]_{x}\right), c\right\} v_{i}(x) \otimes v_{i}(x),
$$

where we have used that $a+(c-a)^{+}=\max \{a, c\}$ for $a, c \geq 0$. In particular, notice that

$$
\lambda_{d(x)}\left[S_{E\left(\mathcal{G}^{\mathrm{op}}\right)}\right]_{x} \geq \max \left\{c, \lambda_{d(x)}\left([A]_{x}\right)\right\}
$$

which implies that the condition number of $\left[S_{E\left(\mathcal{G}^{\circ p}\right)}\right]_{x}$ is smaller than or equal to the condition number of $[A]_{x}=\left[S_{E(\mathcal{F})_{\mathcal{V}}^{\#}}\right]_{x}$ - both acting on $J_{\mathcal{V}}(x)$ - for a.e. $x \in \mathbb{T}^{k}$. That is, the optimal oblique dual $E\left(\mathcal{G}^{\text {op }}\right)$ improves the (spectral) stability of the canonical oblique dual $E(\mathcal{F})_{\mathcal{V}}^{\#}=E\left(\mathcal{F}_{\mathcal{V}}^{\#}\right)$.

The representation of $\left[S_{E\left(\mathcal{G}^{\text {op }}\right)}\right]_{x}$ above motivates the following construction, which also characterizes all elements of $\mathcal{D}_{\mathcal{V}, w}^{S G}(\mathcal{F})$ which are minimal in the sense of Theorem 5.7 .

Definition 5.9 (Non-commutative water-filling at level $c$ in $U_{\mathcal{V}}(E(\mathcal{G}))$ ). Let $\mathcal{G}=\left\{g_{i}\right\}_{i \in \mathbb{I}_{n}}$ be such that $E(\mathcal{G})$ is a frame for $\mathcal{V}$ with frame operator $A=S_{E(\mathcal{G})}$. By Lemma 3.2 we can consider measurable vector fields $v_{j}: \mathbb{T}^{k} \rightarrow \ell^{2}\left(\mathbb{Z}^{k}\right)$ for $j \in \mathbb{I}_{n}$ such that

$$
[A]_{x}=\sum_{j \in \mathbb{I}_{d(x)}} \lambda_{j}\left([A]_{x}\right) v_{j}(x) \otimes v_{j}(x)
$$

is a spectral representation of $[A]_{x}$, where $\left\{v_{j}(x)\right\}_{j \in \mathbb{I}_{d(x)}}$ is an ONB of $J_{\mathcal{V}}(x)$ (here $d(x)=\operatorname{dim} J_{\mathcal{V}}(x) \leq$ $n)$, for a.e. $x \in \mathbb{T}^{k}$.

Given $c \geq 0$ then we define the (non-commutative) water-filling of $A$ at level $c$ with respect to the representation in Eq. (47), denoted $A_{c} \in U_{\mathcal{V}}(E(\mathcal{G}))$, as the unique positive SP operator such that operator $R\left(A_{c}\right) \subset \mathcal{V}$ and

$$
\left[A_{c}\right](x) \stackrel{\text { def }}{=}\left[A_{c}\right]_{x}=\sum_{i \in \mathbb{I}_{r(x)}} \lambda_{i}\left([A]_{x}\right) v_{i}(x) \otimes v_{i}(x)+\sum_{i=r(x)+1}^{d(x)} \max \left\{\lambda_{i}\left([A]_{x}\right), c\right\} v_{i}(x) \otimes v_{i}(x)
$$

where $r(x)=\max \{2 d(x)-n, 0\}\left(\right.$ recall $\mathbb{I}_{0}=\emptyset$ ) for a.e. $x \in \mathbb{T}^{k}$.

Remark 5.10. With the notations of Definition 5.9 . 
1. We point out that $\left[A_{c}\right]$ as described in Eq. (48) is a well defined measurable field of positive semidefinite operators that is essentially bounded.

2. Notice that in the spectral representation of $\left[A_{c}\right](x)$ given in Eq. (48), the eigenvalues are not necessarily arranged in non-increasing order.

3. Finally notice that $A_{c} \in U_{\mathcal{V}}(E(\mathcal{G}))$, since $\left[A_{c}\right](x)-[A]_{x}$ is a positive operator with rank at most $d(x)-r(x) \leq n-d(x)$, for a.e. $x \in \mathbb{T}^{k}$.

We end this section with the following comments: with the notions and notations of Theorem 5.7. let $A=S_{E(\mathcal{F})_{\nu}^{\#}}$ and consider measurable vector fields $v_{i}: \mathbb{T}^{k} \rightarrow \ell^{2}\left(\mathbb{Z}^{k}\right)$, for $i \in \mathbb{I}_{n}$, such that

$$
[A]_{x}=\sum_{i \in \mathbb{I}_{d(x)}} \lambda_{i}\left([A]_{x}\right) v_{i}(x) \otimes v_{i}(x)
$$

is a spectral representation of $[A]_{x}$ with respect to an eigen-basis $\left\{v_{i}(x)\right\}_{i \in \mathbb{I}_{d(x)}}$, where $d(x)=$ $\operatorname{dim}\left(J_{\mathcal{V}}(x)\right)$, for a.e. $x \in \mathbb{T}^{k}$. Let $c>0$ be such that, if $A_{c}$ is the water-filling of $A$ at level $c$ with respect to the representation in Eq. (49) then,

$$
\int_{\mathbb{T}^{k}} \operatorname{tr}\left(\left[A_{c}\right](x)\right) d x=w .
$$

By construction $A_{c} \in U_{\mathcal{V}}\left(E(\mathcal{F})_{\mathcal{V}}^{\#}\right)$ and therefore, by Proposition 4.10, there exists $\mathcal{G}_{0} \in \mathcal{D}_{\mathcal{V}, w}^{S G}(\mathcal{F})$ such that $S_{\mathcal{G}_{0}}=A_{c}$. As we have already noticed, $\mathcal{G}^{\text {op }}$ from Theorem 5.7 is constructed in this way; hence, in this case we have that for every non-decreasing $\varphi \in \operatorname{Conv}\left(\mathbb{R}^{+}\right)$,

$$
P_{\varphi}^{\mathcal{V}}\left(E\left(\mathcal{G}_{0}\right)\right) \leq P_{\varphi}^{\mathcal{V}}(E(\mathcal{G})), \quad \text { for every } \mathcal{G} \in \mathcal{D}_{\mathcal{V}, w}^{S G}(\mathcal{F})
$$

Moreover, by Corollary [5.8, any structural optimal frame $\mathcal{G} \in \mathcal{D}_{\mathcal{V}, w}^{S G}(\mathcal{F})$ (i.e. such that $\mathcal{G}$ is a $P_{\varphi}^{\mathcal{V}}$-minimizer in $\mathcal{D}_{\mathcal{V}, w}^{S G}(\mathcal{F})$ for every $\varphi \in \operatorname{Conv}\left(\mathbb{R}^{+}\right)$) is obtained in this way. That is, the structural optimal SG $\mathcal{V}$-dual frames for $E(\mathcal{F})$ with norm restrictions are exactly those $\mathcal{G} \in \mathcal{D}_{\mathcal{V}, w}^{S G}(\mathcal{F})$ for which their frame operators are obtained in terms of the non-commutative water-filling construction from Definition 5.9.

\section{Appendix: spectral structure of $U_{\mathcal{V}}(E(\mathcal{G}))$}

In what follows we consider a measure space $(X, \mathcal{X}, \rho)$, such that $X \subset \mathbb{T}^{k}$ is a Lebesgue measurable set, $\mathcal{X}$ denotes the $\sigma$-algebra of Lebesgue sets in $X$ and $\rho$ is the Lebesgue measure restricted to $\mathcal{X}$.

Proposition 6.1. Let $G: X \rightarrow \mathcal{M}_{n}(\mathbb{C})^{+}$be a bounded measurable field of positive semidefinite matrices with associated measurable eigenvalues $\lambda_{j}: X \rightarrow \mathbb{R}^{+}$for $j \in \mathbb{I}_{n}$ such that $\lambda_{1} \geq \ldots \geq \lambda_{n}$. Assume that the measurable functions $\beta_{j}: X \rightarrow \mathbb{R}^{+}$for $j \in \mathbb{I}_{n-1}$ satisfy the interlacing conditions

$$
\lambda_{j}(x) \geq \beta_{j}(x) \geq \lambda_{j+1}(x) \quad \text { x-a.e. for } j \in \mathbb{I}_{n-1} .
$$

Then there exists a measurable map $W: X \rightarrow \mathcal{M}_{n, n-1}(\mathbb{C})$ such that $W^{*}(x) W(x)=I_{n-1}$ and

$$
\lambda\left(W^{*}(x) G(x) W(x)\right)=\left(\beta_{1}(x), \ldots, \beta_{n-1}(x)\right), \quad \text { for a.e. } \quad x \in X .
$$

Proof. We argue by induction on $n$ (the size of $G$ ). Notice that $\beta_{1} \geq \ldots \geq \beta_{n-1}$ by Eq. (50). Using the results of [43], we can consider measurable vector fields $u_{j}: X \rightarrow \mathbb{C}^{n}$ for $j \in \mathbb{I}_{n}$ such that $\left\{u_{j}(x)\right\}_{j \in \mathbb{I}_{n}}$ is an ONB of eigenvectors of $G(x)$ for a.e. $x \in X$. 
Assume first that $\beta_{n-1}=\lambda_{n}$. Set $G^{\prime}(x)=V(x)^{*} G(x) V(x)$ where $V(x)$ is the $n \times(n-1)$ matrix whose columns are the vectors $u_{1}(x), \ldots, u_{n-1}(x)$, for $x \in X$. Then, $G^{\prime}$ is a bounded measurable field of (diagonal) positive semidefinite matrices of size $n-1$ with measurable eigenvalues $\lambda_{j}: X \rightarrow$ $\mathbb{R}^{+}$for $j \in \mathbb{I}_{n-1}$. If we assume that we can find a measurable function $Z: X \rightarrow \mathcal{M}_{n-1, n-2}(\mathbb{C})$ such that $Z^{*}(x) Z(x)=I_{n-2}$ and $\lambda\left(Z^{*}(x) G^{\prime}(x) Z(x)\right)=\left(\beta_{1}(x), \ldots, \beta_{n-2}(x)\right)$ for a.e. $x \in X$, we let

$$
W(x)=\left(\begin{array}{cc}
Z(x) & 0_{n-1} \\
0_{n-2}^{t} & 1
\end{array}\right), \quad \text { for } x \in X .
$$

Then, it is easy to see that $W: X \rightarrow \mathcal{M}_{n, n-1}(\mathbb{C})$ has the desired properties. By iterating the previous argument and considering a convenient partition of $X$ into measurable sets, we can assume without loss of generality that

$$
\lambda_{j}(x)>\beta_{j}(x)>\lambda_{j+1}(x), \quad \text { for a.e. } x \in X, \quad j \in \mathbb{I}_{n-1} .
$$

In this case we set

$$
\gamma_{j}(x)=\frac{\prod_{i \in \mathbb{I}_{n-1}}\left(\lambda_{j}(x)-\beta_{i}(x)\right)}{\prod_{k \neq j}\left(\lambda_{j}(x)-\lambda_{k}(x)\right)}, \quad \text { for } x \in X, \quad j \in \mathbb{I}_{n} .
$$

The previous assumptions (strict interlacing inequalities) imply that $\gamma_{j}(x)>0$ is defined for a.e. $x \in X$; moreover, the functions $\gamma_{j}: X \rightarrow \mathbb{R}^{+}$are measurable for $j \in \mathbb{I}_{n}$.

Set $\xi_{j}=\gamma_{j}^{1 / 2}: X \rightarrow \mathbb{R}^{+}$for $j \in \mathbb{I}_{n}$, let $v=\sum_{j \in \mathbb{I}_{n}} \xi_{j} u_{j}: X \rightarrow \mathbb{C}^{n}$ and let $P: X \rightarrow \mathcal{M}_{n}(\mathbb{C})^{+}$ given by $P(x)=I-P_{v(x)}$ (the orthogonal projection onto $\{v(x)\}^{\perp}$, notice that $v(x) \neq 0$ a.e.). Let $p_{x}(t) \in \mathbb{R}[t]$ denote the characteristic polynomial of $P(x) G(x) P(x)$. Then, a well known argument in terms of alternate tensor products (see [4]) shows that

$$
p_{x}(t)=t \sum_{j \in \mathbb{I}_{n}} \gamma_{j}(x) \prod_{k \neq j}\left(t-\lambda_{k}(x)\right) \Longrightarrow p_{x}\left(\lambda_{j}(x)\right)=\lambda_{j}(x) \prod_{i \in \mathbb{I}_{n-1}}\left(\lambda_{j}(x)-\beta_{i}(x)\right)
$$

for a.e. $x \in X, j \in \mathbb{I}_{n}$ and $p_{x}(0)=0$. Therefore,

$$
p_{x}(t)=t \prod_{j \in \mathbb{I}_{n-1}}\left(t-\beta_{j}\right) \quad \text { and } \quad \sum_{j \in \mathbb{I}_{n}} \xi_{j}^{2}(x)=1, \quad \text { for a.e. } x \in X,
$$

by comparing the leading coefficients of the two representations of the polynomial. This last normalization condition shows, in particular, that $P(x)=I-v(x) \otimes v(x)$ for $x \in X$ a.e. and hence $P$ is a measurable function.

Finally, let $\left\{w_{j}: X \rightarrow \mathbb{C}^{n}\right\}_{j \in \mathbb{I}_{n}}$ be a measurable ONB of eigenvectors functions for $P$ such that $P(x) w_{n}(x)=0$ for a.e. $x \in X$. Set $W: X \rightarrow \mathcal{M}_{n, n-1}(\mathbb{C})$ such that $W(x)$ is the $n \times n-1$ matrix whose columns are the vectors $w_{1}(x), \ldots, w_{n-1}(x)$; then $W$ is a measurable function with the desired properties.

Lemma 6.2. Let $\lambda_{j}: X \rightarrow \mathbb{R}^{+}$for $j \in \mathbb{I}_{n}$ be measurable functions such that $\lambda_{1} \geq \ldots \geq \lambda_{n}$. Let $d \in \mathbb{I}_{n-1}$ and let $\beta_{j}: X \rightarrow \mathbb{R}^{+}$for $j \in \mathbb{I}_{d}$ be measurable functions such that $\beta_{1} \geq \ldots \geq \beta_{d}$ and such that they satisfy the interlacing inequalities

$$
\lambda_{j}(x) \geq \beta_{j}(x) \geq \lambda_{n-d+j}(x), \quad \text { for .a.e. } x \in X, \quad j \in \mathbb{I}_{d} .
$$

Then, there exist measurable functions $\gamma_{i, j}: X \rightarrow \mathbb{R}^{+}$for $0 \leq i \leq n-d$ and $j \in \mathbb{I}_{n-i}$ such that:

1. $\gamma_{0, j}=\lambda_{j}$ for $j \in \mathbb{I}_{n}$ and $\gamma_{n-d, j}=\beta_{j}$ for $j \in \mathbb{I}_{d}$; 
2. For $0 \leq i \leq n-d$ then $\gamma_{i, j}(x) \geq \gamma_{i, j+1}(x)$ for $j \in \mathbb{I}_{n-i-1}$, for a.e. $x \in X$;

3. For $0 \leq i \leq n-d-1$ then $\gamma_{i, j}(x) \geq \gamma_{i+1, j}(x) \geq \gamma_{i, j+1}(x)$ for $j \in \mathbb{I}_{n-i-1}$, for a.e. $x \in X$.

Proof. We argue by (decreasing) induction in terms of $d$. Notice that the statement is trivially true if $d=n-1$. Assume that the result is true for $d+1$ interlacing measurable functions for some $d \in \mathbb{I}_{n-2}$. Given the measurable functions $\beta_{j}$ for $j \in \mathbb{I}_{d}$ as above, we shall construct measurable functions $\alpha_{j}: X \rightarrow \mathbb{R}^{+}$for $j \in \mathbb{I}_{d+1}$ such that

$$
\lambda_{j} \geq \alpha_{j} \geq \lambda_{n-(d+1)+j} \quad \text { for } \quad j \in \mathbb{I}_{d+1} \quad \text { and } \quad \alpha_{j} \geq \beta_{j} \geq \alpha_{j+1} \quad \text { for } \quad j \in \mathbb{I}_{d},
$$

and hence $\alpha_{1} \geq \ldots \geq \alpha_{d+1}$. Notice that the lemma would be a consequence of this construction and the inductive hypothesis (where the maps $\alpha_{j}$ play the role of $\gamma_{n-d+1, j}$ for $j \in \mathbb{I}_{d+1}$ ).

First notice that by the interlacing inequalities in Eq. (52) we have that

$$
\min \left\{\lambda_{r+1}, \beta_{r}\right\} \geq \max \left\{\beta_{r+1}, \lambda_{n-d+r}\right\}, \quad \text { for } r \in \mathbb{I}_{d-1} \text { and for a.e. } x \in X .
$$

We define $\alpha_{j}: X \rightarrow \mathbb{R}^{+}$, for $j \in \mathbb{I}_{d+1}$, as follows:

$$
\alpha_{j}:=\left\{\begin{array}{ccc}
\max \left\{\beta_{j}, \lambda_{n-(d+1)+j}\right\} & \text { if } & 1 \leq j \leq d \\
\min \left\{\beta_{d}, \lambda_{d+1}\right\} & \text { if } & j=d+1
\end{array}\right.
$$

By construction the functions $\alpha_{j}$ are measurable, and it is easy to check (by using Eq. (54)) that they satisfy Eq. (53).

The following result is the Fan-Pall interlacing inequalities theorem for measurable fields of positive operators.

Theorem 6.3. Let $G: X \rightarrow \mathcal{M}_{n}(\mathbb{C})^{+}$be a bounded measurable field of positive semidefinite matrices with associated measurable eigenvalues $\lambda_{j}: X \rightarrow \mathbb{R}^{+}$for $j \in \mathbb{I}_{n}$ such that $\lambda_{1} \geq \ldots \geq \lambda_{n}$. Let $d \in \mathbb{I}_{n-1}$ and let $\beta_{j}: X \rightarrow \mathbb{R}^{+}$for $j \in \mathbb{I}_{d}$ be measurable functions such that $\beta_{1} \geq \ldots \geq \beta_{d}$. Then the following conditionas are equivalent:

1. $\lambda_{j}(x) \geq \beta_{j}(x) \geq \lambda_{n-d+j}(x)$, for a.e. $x \in X, \quad j \in \mathbb{I}_{d}$.

2. There exists a projection valued measurable function $P: X \rightarrow \mathcal{M}_{n}(\mathbb{C})^{+}$such that

$$
\operatorname{rk} P(x)=d \quad \text { and } \quad \lambda(P(x) G(x) P(x))=\left(\beta_{1}(x), \ldots, \beta_{d}(x), 0_{n-d}\right), \quad \text { for a.e. } x \in X .
$$

Proof. Assume first that the functions $\left\{\beta_{j}\right\}_{j \in \mathbb{I}_{d}}$ satisfy the interlacing inequalities in item 1. Let $\gamma_{i, j}: X \rightarrow \mathbb{R}^{+}$for $0 \leq i \leq n-d$ and $j \in \mathbb{I}_{n-i}$ be measurable functions as in By Lemma 6.2. By Proposition 6.1 there exists a measurable function $W_{1}: X \rightarrow \mathcal{M}_{n, n-1}(\mathbb{C})$ such that

$$
W_{1}(x)^{*} W_{1}(x)=I_{n-1} \quad \text { and } \quad \lambda\left(W_{1}(x)^{*} G W_{1}(x)\right)=\left(\gamma_{1,1}(x), \ldots, \gamma_{1, n-1}(x)\right), \quad \text { for a.e. } x \in X .
$$

Arguing as before, using Proposition 6.1 we can construct for $2 \leq i \leq n-d$ measurable functions $W_{i}: X \rightarrow \mathcal{M}_{n-i+1, n-i}(\mathbb{C})$ such that $W_{i}(x)^{*} W_{i}(x)=I_{n-i}$ for a.e. $x \in X$ and

$$
\lambda\left(W_{i}(x)^{*} \cdots W_{1}(x)^{*} G(x) W_{1}(x) \cdots W_{i}(x)\right)=\left(\gamma_{i, 1}(x), \ldots, \gamma_{i, n-i}(x)\right), \quad \text { for a.e. } x \in X .
$$

Let $W=W_{1} \cdots W_{n-d}: X \rightarrow \mathcal{M}_{n, d}(\mathbb{C})$ which is measurable by construction and notice that

$$
W^{*}(x) W(x)=I_{d} \quad \text { and } \quad \lambda\left(W(x)^{*} G(x) W(x)\right)=\left(\beta_{1}(x), \ldots, \beta_{d}(x)\right), \quad \text { for a.e. } x \in X .
$$

Hence, if we set $P=W W^{*}: X \rightarrow \mathcal{M}_{n}(\mathbb{C})$ then $P$ is a measurable field of projections with the desired properties.

Conversely, assume that there exists a projection valued measurable function $P: X \rightarrow \mathcal{M}_{n}(\mathbb{C})^{+}$ satisfying item 2 . Then item 1 is a straightforward consequence of the so-called Cauchy interlacing inequalities from matrix analysis (see for example [4]). 
Let $\mathcal{G}=\left\{g_{i}\right\}_{i \in \mathbb{I}_{n}}$ be such that $E(\mathcal{G})$ is a frame for the SI subspace $\mathcal{V}$, with frame operator $A=S_{E(\mathcal{G})}$. Recall that (see Definition 4.9)

$$
U_{\mathcal{V}}(E(\mathcal{G}))=\left\{A+B: B \in L\left(L^{2}\left(\mathbb{R}^{k}\right)\right)^{+} \text {is } \mathrm{SP}, R(B) \subset \mathcal{V}, \operatorname{rk}\left([B]_{x}\right) \leq n-d(x), \text { for a.e. } x \in \mathbb{T}^{k}\right\} .
$$

Using the Fan-Pall inequalities for measurable fields of matrices we can now describe the fine spectral structure of the elements in $U_{\mathcal{V}}(E(\mathcal{G}))$

Theorem 6.4. Let $\mathcal{V}$ be a SI subspace in $L^{2}\left(\mathbb{R}^{k}\right)$ with $\operatorname{Spec}(\mathcal{V}) \subseteq X$ and let $\mathcal{G}=\left\{g_{i}\right\}_{i \in \mathbb{I}_{n}}$ be such that $E(\mathcal{G})$ is a frame for $\mathcal{V}$ with frame operator $A=S_{E(\mathcal{G})}$. Let $d: X \rightarrow \mathbb{N}$ be the measurable function given by $d(x)=\operatorname{dim} J_{\mathcal{V}}(x)$, for $x \in \operatorname{Spec}(\mathcal{V})$, and let $m(\cdot)=2 d(\cdot)-n$. Given a measurable function $\mu: X \rightarrow\left(\ell_{+}^{1}(\mathbb{N})^{+}\right)^{\downarrow}$ (decreasing sequences) the following are equivalent:

1. There exists $C \in U_{\mathcal{V}}(E(\mathcal{G}))$ such that $\mu(x)=\lambda\left([A]_{x}\right)$ for a.e. $x \in X$;

2. $\mu(x)=0$ for every $x \notin \operatorname{Spec}(\mathcal{V})$. If $x \in \operatorname{Spec}(\mathcal{V})$ then $\mu_{i}(x)=0$ for $i \geq d(x)+1$ and

(a) in case that $m(x) \leq 0$, then $\mu_{i}(x) \geqslant \lambda_{i}\left([A]_{x}\right)$ for $i \in \mathbb{I}_{d(x)}$;

(b) in case that $m(x) \geq 1$, then $\mu_{i}(x) \geqslant \lambda_{i}\left([A]_{x}\right)$ for $i \in \mathbb{I}_{d(x)}$ and

$$
\mu_{n-d(x)+i}(x)=\mu_{d(x)-m(x)+i}(x) \leq \lambda_{i}\left([A]_{x}\right) \quad \text { for } \quad i \in \mathbb{I}_{m(x)} .
$$

Proof. First notice that by considering a convenient finite partition of $X$ into measurable sets we can assume, without loss of generality, that $d(x)=d \in \mathbb{N}$ for a.e. $x \in \operatorname{Spec}(\mathcal{V})$.

Let $C \in U_{\mathcal{V}}(E(\mathcal{G}))$, and assume that $\mu=\lambda([C])$. By hypothesis, there exists $B \in L\left(L^{2}\left(\mathbb{R}^{k}\right)\right)^{+} \mathrm{SP}$, with $R(B) \subset \mathcal{V}, \operatorname{rk}\left([B]_{x}\right) \leq n-d(x)$ for a.e. $x \in \mathbb{T}^{k}$, such that $C=A+B$. By Lemma 4.8 there exists $\mathcal{Z}=\left\{z_{i}\right\}_{i \in \mathbb{I}_{n}} \in \mathcal{V}^{n}$ such that $T_{E(\mathcal{G})} T_{E(\mathcal{Z})}^{*}=0$ and $B=S_{E(\mathcal{Z})}$. If we let $\mathcal{G}+\mathcal{Z}=\left\{g_{i}+z_{i}\right\}_{i \in \mathbb{I}_{n}}$ then $T_{E(\mathcal{G}+\mathcal{Z})}=T_{E(\mathcal{G})}+T_{E(\mathcal{Z})}$ and

$$
S_{E(\mathcal{G}+\mathcal{Z})}=T_{E(\mathcal{G}+\mathcal{Z})} T_{E(\mathcal{G}+\mathcal{Z})}^{*}=S_{E(\mathcal{G})}+S_{E(\mathcal{Z})}=A+S_{E(\mathcal{Z})}=C
$$

with $R\left([C]_{x}\right)=J_{\mathcal{V}}(x)$ and $\operatorname{dim} J_{\mathcal{V}}(x)=d \leq n$. Then,

$$
\lambda_{j}\left(\left[T_{E(\mathcal{G}+\mathcal{Z})}^{*} T_{E(\mathcal{G}+\mathcal{Z})}\right]_{x}\right)=\mu_{j}(x) \quad \text { for } j \in \mathbb{I}_{d} \text { and a.e. } x \in \operatorname{Spec}(\mathcal{V}) .
$$

Moreover, if we let $P: \operatorname{Spec}(\mathcal{V}) \rightarrow \mathcal{M}_{n}(\mathbb{C})^{+}$be the projection valued measurable function such that $P(x)$ is the orthogonal projection onto span $\{\Gamma \mathcal{G}(x)\}=R\left(T_{\Gamma \mathcal{G}(x)}^{*}\right)$ then, using again that $T_{E(\mathcal{G})} T_{E(\mathcal{Z})}^{*}=0$ we see that

$$
P(x)\left(\left[T_{E(\mathcal{G}+\mathcal{Z})}^{*} T_{E(\mathcal{G}+\mathcal{Z})}\right]_{x}\right) P(x)=\left[T_{E(\mathcal{G})}^{*} T_{E(\mathcal{G})}\right]_{x}, \quad \text { for a.e. } x \in \operatorname{Spec}(\mathcal{V}) .
$$

Since $\operatorname{rk}(P(x))=d \leq n$ and

$$
\lambda_{j}\left(\left[T_{E(\mathcal{G})}^{*} T_{E(\mathcal{G})}\right]_{x}\right)=\lambda_{j}\left(\left[T_{E(\mathcal{G})} T_{E(\mathcal{G})}^{*}\right]_{x}\right)=\lambda_{j}\left([A]_{x}\right), \quad \text { for } j \in \mathbb{I}_{d} \text { and a.e. } x \in \operatorname{Spec}(\mathcal{V})
$$

then, using Theorem 6.3 we conclude that that the Fan-Pall inequalities hold between

$$
\left(\mu_{1}(x), \ldots, \mu_{d}(x), 0_{n-d}\right) \quad \text { and } \quad\left(\lambda_{1}\left([A]_{x}\right), \ldots, \lambda_{d}\left([A]_{x}\right), 0_{n-d}\right) .
$$

A careful inspection of these inequalities for the previous vectors shows that the inequalities in items 2.a and 2.b. above hold (according to the relation between $n$ and $d$ ).

Conversely, let $\mu: \operatorname{Spec}(\mathcal{V}) \rightarrow \ell^{1}(\mathbb{N})^{+}$satisfy the conditions in item 2 and let $D_{\mu}(\cdot): \operatorname{Spec}(\mathcal{V}) \rightarrow$ $\mathcal{M}_{n}(\mathbb{C})^{+}$be the measurable field of positive semidefinite matrices such that $D_{\mu}(x)$ is the diagonal 
matrix with main diagonal $\left(\mu_{1}(x), \ldots, \mu_{d}(x), 0_{n-d}\right)$ for $x \in X$. Then, by Theorem 6.3 there exists a projection valued measurable function $P: \operatorname{Spec}(\mathcal{V}) \rightarrow \mathcal{M}_{n}(\mathbb{C})^{+}$such that

$$
\operatorname{tr}(P(x))=d \quad \text { and } \quad \lambda\left(P(x) D_{\mu}(x) P(x)\right)=\left(\lambda_{1}\left([A]_{x}\right), \ldots, \lambda_{d}\left([A]_{x}\right), 0_{n-d}\right) \in\left(\mathbb{R}^{+}\right)^{n} .
$$

In this case we see that

$$
\lambda\left(D_{\mu}^{1 / 2} P(x) D_{\mu}^{1 / 2}\right)=\left(\lambda_{1}\left([A]_{x}\right), \ldots, \lambda_{d}\left([A]_{x}\right), 0_{n-d}\right) \in\left(\mathbb{R}^{+}\right)^{n} .
$$

Let $D_{\lambda}(x)$ be the diagonal matrix with main diagonal $\left(\lambda_{1}\left([A]_{x}\right), \ldots, \lambda_{d}\left([A]_{x}\right), 0_{n-d}\right)$. By taking an appropriate measurable field of unitary matrices $U(x): \operatorname{Spec}(\mathcal{V}) \rightarrow \mathcal{M}_{n}(\mathbb{C})$ we conclude that

$$
D_{\lambda}(x)=U(x)^{*}\left(D_{\mu}^{1 / 2} P(x) D_{\mu}^{1 / 2}\right) U(x), \quad \text { for a.e. } x \in \operatorname{Spec}(\mathcal{V}) .
$$

Arguing as in the proof of Lemma 4.8 we see that there exist measurable fields of vectors $v_{j}$ : $\operatorname{Spec}(\mathcal{V}) \rightarrow \ell^{2}\left(\mathbb{Z}^{k}\right)$, for $j \in \mathbb{I}_{d}$, such that $[A]_{x} v_{j}(x)=\lambda_{j}\left([A]_{x}\right) v_{j}(x)$ and $\mathcal{B}(x)=\left\{v_{j}(x)\right\}_{i \in \mathbb{I}_{d}}$ is an ONB of $J_{\mathcal{V}}(x)$ for a.e. $x \in \operatorname{Spec}(\mathcal{V})$. We finally consider $B \in L\left(L^{2}\left(\mathbb{R}^{k}\right)\right)^{+}$S.P. with $R(B) \subset \mathcal{V}$, uniquely determined by the condition:

$$
\left\{[B]_{x}\right\}_{\mathcal{B}(x)}=U(x)^{*}\left(D_{\mu}^{1 / 2}(I-P(x)) D_{\mu}^{1 / 2}\right) U(x), \quad \text { for a.e. } x \in \operatorname{Spec}(\mathcal{V})
$$

where $\left\{[B]_{x}\right\}_{\mathcal{B}(x)}$ stands for the matrix representation of $[B]_{x}$ with respect to the ONB $\mathcal{B}(x)$ of $J_{\mathcal{V}}(x)$; in particular, using that $\operatorname{rk}(I-P(x))=n-d$, we conclude that $\operatorname{rk}\left([B]_{x}\right) \leq n-d$ for a.e. $x \in \operatorname{Spec}(\mathcal{V})$. On the other hand, by construction of $\mathcal{B}(x)$, we have that $\left\{[A]_{x}\right\}_{\mathcal{B}(x)}=D_{\lambda}(x)$ : thus, using Eqs (56) and (57) we have that

$$
\left\{[A]_{x}+[B]_{x}\right\}_{\mathcal{B}(x)}=\left\{[A]_{x}\right\}_{\mathcal{B}(x)}+\left\{\left[B_{x}\right]\right\}_{\mathcal{B}(x)}=U(x)^{*} D_{\mu} U(x), \quad \text { for a.e. } x \in \operatorname{Spec}(\mathcal{V}) .
$$

This last fact implies $C=A+B \in U_{\mathcal{V}}(E(\mathcal{G}))$ satisfies that $\lambda_{j}\left([C]_{x}\right)=\mu_{j}(x)$ for $j \in \mathbb{N}$ and a.e. $x \in \operatorname{Spec}(\mathcal{V})$.

\section{References}

[1] A. Aldroubi, C. Cabrelli, D. Hardin, U. Molter, Optimal shift invariant spaces and their Parseval frame generators. Appl. Comput. Harmon. Anal. 23 (2007), no. 2, 273-283.

[2] M. Anastasio, C. Cabrelli, Sampling in a union of frame generated subspaces. Sampl. Theory Signal Image Process. 8 (2009), no. 3, 261-286.

[3] J. Antezana, P. Massey, M. Ruiz, D. Stojanoff, The Schur-Horn theorem for operators and frames with prescribed norms and frame operator. Illinois J. Math. 51 (2007), no. 2, 537-560.

[4] R. Bhatia, Matrix Analysis, Berlin-Heildelberg-New York, Springer 1997.

[5] M.J. Benac, P. Massey, D. Stojanoff, Aliasing and oblique dual pair designs for consistent sampling. Linear Algebra Appl. 487 (2015), 112-145.

[6] J.J. Benedetto, M. Fickus, Finite normalized tight frames. Frames. Adv. Comput. Math. 18 (2003), no. 2-4, 357-385.

[7] C. de Boor, R. DeVore, A. Ron, The structure of finitely generated shift-invariant spaces in $L_{2}\left(\mathbf{R}^{d}\right)$. J. Funct. Anal. 119 (1994), no. 1, 37-78.

[8] M. Bownik. The structure of shift-invariant subspaces of $L^{2}\left(\mathbf{R}^{n}\right)$. J. Funct. Anal. 177 (2000), no. 2, 282-309.

[9] M. Bownik, J. Jasper, Characterization of sequences of frame norms. J. Reine Angew. Math. 654 (2011), 219-244.

[10] M. Bownik, J. Jasper, The Schur-Horn theorem for operators with finite spectrum. Trans. Amer. Math. Soc. 367 (2015), no. $7,5099-5140$.

[11] C. Cabrelli, V. Paternostro, Shift-invariant spaces on LCA groups. J. Funct. Anal. 258 (2010), no. 6, 2034-2059. 
[12] J. Cahill, M. Fickus, D. Mixon, M. Poteet, N. Strawn, Constructing finite frames of a given spectrum and set of lengths. Appl. Comput. Harmon. Anal. 35 (2013), no. 1, 52-73.

[13] P.G. Casazza, M. Fickus, J. Kovacevic, M.T. Leon, J.C. Tremain, A physical interpretation of tight frames. Harmonic analysis and applications, 51-76, Appl. Numer. Harmon. Anal., Birkhäuser Boston, MA, 2006.

[14] P.G. Casazza, M. Leon, Existence and Construction of Finite Frames with a Given Frame Operator, International Journal of Pure and Applied Mathematics, Vol. 63, No. 2 (2010), p. 149-158.

[15] K.M. Chong, Doubly stochastic operators and rearrangement theorems. J. Math. Anal. Appl. 56 (1976), no. 2, 309-316.

[16] O. Christensen, An introduction to frames and Riesz bases. Applied and Numerical Harmonic Analysis. Birkhäuser Boston, Inc., Boston, MA, 2003.

[17] O. Christensen, Y.C. Eldar, Oblique dual frames and shift-invariant spaces. Appl. Comput. Harmon. Anal. 17 (2004), no. $1,48-68$.

[18] O. Christensen, Y.C. Eldar, Characterization of oblique dual frame pairs. EURASIP J. Appl. Signal Process. (2006), 1-11.

[19] T.M. Cover, J.A. Thomas, Joy A. Elements of information theory. Second edition. Wiley-Interscience [John Wiley \& Sons], Hoboken, NJ, 2006.

[20] F. Deutsch, The angle between subspaces of a Hilbert space. Approximation theory, wavelets and applications (Maratea, 1994), 107-130, NATO Adv. Sci. Inst. Ser. C Math. Phys. Sci., 454, 1995.

[21] D. E. Dutkay, The local trace function of shift invariant subspaces, J. Operator Theory 52 (2004), $267-291$.

[22] Y.C. Eldar, Sampling with arbitrary sampling and reconstruction spaces and oblique dual frame vectors. J. Fourier Anal. Appl. 9 (2003), no. 1, 77-96.

[23] Y.C. Eldar, T. Werther, General framework for consistent sampling in Hilbert spaces. Int. J. Wavelets Multiresolut. Inf. Process. 3 (2005), no. 4, 497-509.

[24] M. Fickus, D. G. Mixon and M. J. Poteet, Frame completions for optimally robust reconstruction, Proceedings of SPIE, 8138: 81380Q/1-8 (2011).

[25] D. Han, Frame representations and Parseval duals with applications to Gabor frames. Trans. Amer. Math. Soc. 360 (2008), no. $6,3307-3326$.

[26] C. Heil, Y.Y. Koo, J.K. Lim, Duals of frame sequences, Acta Appl. Math. 107 (2009), No. 1-3 , 75-90.

[27] H. Helson, Lectures on Invariant Subspaces, Academic Press, New York - London, 1964.

[28] A.A. Hemmat, J.P. Gabardo. The uniqueness of shift-generated duals for frames in shift-invariant subspaces. J. Fourier Anal. Appl. 13 (2007), no. 5, 589-606.

[29] A.A. Hemmat, J.P. Gabardo, Properties of oblique dual frames in shift-invariant systems. J. Math. Anal. Appl. 356 (2009), no. $1,346-354$

[30] A.J.E.M. Janssen, The Zak transform and sampling theorems for wavelet subspaces, IEEE Trans. Signal Processing, 41 (1993), 3360-3364.

[31] V. Kaftal, G. Weiss, An infinite dimensional Schur-Horn theorem and majorization theory. J. Funct. Anal. 259 (2010), no. $12,3115-3162$.

[32] H. O. Kim, R. Y. Lim and J. K. Lim, The infimum cosine angle between two finitely generated shift-invariant spaces and its applications. Appl. Comput. Harmon. Anal. 19 (2005), no. 2, 253-281.

[33] H. O. Kim, R. Y. Lim and J. K. Lim, Characterization of the closedness of the sum of two shift-invariant spaces, J. Math. Anal. Appl., 320 (2006), 381-395.

[34] A.W. Marshall, I. Olkin, B.C. Arnold, Inequalities: theory of majorization and its applications. Second edition. Springer Series in Statistics. Springer, New York, 2011.

[35] P. Massey, M. Ruiz, Tight frame completions with prescribed norms, Samp. Theory in Sig. and Image Proccesing 7(1) (2008), 1-13.

[36] P. Massey, M. Ruiz, Minimization of convex functionals over frame operators. Adv. Comput. Math. 32 (2010), no. 2, 131-153.

[37] P. Massey, M. Ruiz, D. Stojanoff, Optimal dual frames and frame completions for majorization. Appl. Comput. Harmon. Anal. 34 (2013), no. 2, 201-223.

[38] P. Massey, M. Ruiz, D. Stojanoff, Optimal Frame Completions with Prescribed Norms for Majorization. J. Fourier Anal. Appl. 20 (2014), no. 5, 1111-1140. 
[39] P. Massey, M. Ruiz, D. Stojanoff, Optimal frame completions. Adv. Comput. Math. 40 (2014), no. 5-6, 1011-1042.

[40] P. Massey, M. Ruiz, D. Stojanoff, Multiplicative Lidskii's inequalities and optimal perturbations of frames, Linear Algebra Appl. 469 (2015), 539-568.

[41] D.P. Palomar,J.R. Fonollosa, Practical algorithms for a family of waterfilling solutions. IEEE Trans. Signal Process. 53 (2005), no. 2, part 1, 686-695.

[42] M. J. Poteet, Parametrizing finite frames and optimal frame completions, Doctoral thesis, Graduate School of Engineering and Management, Air Force Institute of Technology, Air University.

[43] A. Ron, Z. Shen, Frames and stable bases for shift-invariant subspaces of $L_{2}\left(\mathbb{R}^{d}\right)$. Canad. J. Math. 47 (1995), no. 5 , 1051-1094.

[44] John V. Ryff, Orbits of $L^{1}$-functions under doubly stochastic transformations. Trans. Amer. Math. Soc. 117 (1965) 92-100.

[45] G. Scutari, D.P. Palomar, S. Barbarossa, The MIMO iterative waterfilling algorithm. IEEE Trans. Signal Process. 57 (2009), no. 5, 1917-1935.

[46] C.E. Shannon, Communication in the presence of noise. Proc. I.R.E. 37, (1949). 10-21.

[47] I. E. Telatar, Capacity of multi-antenna Gaussian channels, Eur. Trans. Telecommun., vol. 10, no. 6, pp. 585-595, Nov-Dec. 1999.

[48] M. Unser, A. Aldroubi, A general sampling theory for nonideal acquisition devices, IEEE Trans Signal Processing, vol. 42 (1994) no. 11, 2915-2925.

[49] M. Unser, J. Zerubia, Generalized sampling: Stability and performance analysis, IEEE Trans. Signal Processing, 45 (1997), 2941-2950. 